\title{
Separable Dorsal Raphe Dopamine Projections Mediate Sociability and Valence
}

Kay Tye ( $\nabla$ tye@salk.edu )

Salk Institute https://orcid.org/0000-0002-2435-0182

\section{Gillian Matthews}

https://orcid.org/0000-0001-6754-0333

Mackenzie Lemieux

Salk Institute

\section{Elizabeth Brewer}

Salk Institute

Raymundo Miranda

Salk Institute

Matilde Borio

Salk Institute

\section{Enzo Peroni}

Institut Pasteur https://orcid.org/0000-0001-7567-1499

\section{Laurel Keyes}

Salk Institute

Grace Pereira

Salk Institute

Alba López Moraga

Salk Institute https://orcid.org/0000-0002-2084-4855

Eyal Kimchi

Salk Institute

\section{Nancy Padilla-Coreano}

Salk Institute for Biological Studies https://orcid.org/0000-0001-9293-2697

\section{Romy Wichmann}

Salk Institute

\section{Anna Palle}

Salk Institute

Christopher Lee

Salk Institute 
Keywords:

Posted Date: December 3rd, 2021

DOI: https://doi.org/10.21203/rs.3.rs-1025403/v1

License: (c) (1) This work is licensed under a Creative Commons Attribution 4.0 International License. Read Full License 
1 Separable Dorsal Raphe Dopamine Projections Mediate Sociability and Valence

$3 \quad 1,2,3,{ }^{*}$ Gillian A. Matthews, ${ }^{1,2,3}$ Mackenzie E. Lemieux, ${ }^{2}$ Elizabeth M. Brewer, ${ }^{1,2}$ Matilde Borio, ${ }^{1,2}$ Raymundo 4 Miranda, ${ }^{1}$ Laurel Keyes, ${ }^{2}$ Enzo Peroni, ${ }^{2}$ Grace S. Pereira, ${ }^{2}$ Alba Lopez Moraga, ${ }^{2}$ Anna Pallé, $5 \quad{ }^{1,4}$ Christopher R. Lee, ${ }^{2}$ Eyal Y. Kimchi, ${ }^{1,2}$ Nancy Padilla-Coreano, ${ }^{1,2}$ Romy Wichmann, ${ }^{1,2,4,}{ }^{*}$ Kay M. Tye

7 'Salk Institute for Biological Studies, 10010 N Torrey Pines Rd, La Jolla, CA 92037, USA.

8 2The Picower Institute for Learning and Memory, Department of Brain and Cognitive Sciences, 9 Massachusetts Institute of Technology, Cambridge, MA 02139, USA.

$10{ }^{3}$ Co-first author.

$11{ }^{4}$ Neurosciences Graduate Program, University of California San Diego, La Jolla, CA 92093, USA

$12{ }^{*}$ To Whom Correspondence Should be Addressed: g.a.c.matthews@gmail.com (G.A.M.), 13 tye@salk.edu (K.M.T.). 


\section{Abstract}

Affiliative social connections facilitate well-being and survival in numerous species. Engaging in social interactions requires positive and negative motivational drive, elicited through coordinated activity across neural circuits. However, the identity, interconnectivity, and functional encoding of social information within these circuits remains poorly understood. Here, we focused on downstream projections of dorsal raphe nucleus (DRN) dopamine neurons (DRNDAT), which we previously implicated in 'negative drive'-induced social motivation. We show that three prominent DRNDAT projections - to the bed nucleus of the stria terminalis (BNST), central amygdala (CeA), and posterior basolateral amygdala $(B L P)$ - play separable roles in behavior, despite substantial collateralization. Photoactivation of the DRN ${ }^{\text {DAT }}$-CeA projection promoted social behavior and photoactivation of the DRNDAT-BNST projection promoted exploratory behavior, while the DRNDAT-BLP projection supported place avoidance, suggesting a negative affective state. Downstream regions showed diverse, region-specific, receptor expression, poising DRNDAT neurons to act through dopamine, neuropeptide, and glutamate transmission. Furthermore, we show ex vivo that the effect of DRNDAT photostimulation on downstream neuron excitability was predicted by baseline cell properties, suggesting cell-type-specific modulation. Collectively, these data indicate that DRNDAT neurons may bias behavior via precise modulation of cellular activity in broadly-distributed target structures. 
A close social network confers a survival advantage, both in the wild and in the laboratory ${ }^{1-3}$. In humans, objective or subjective isolation (loneliness) increases mortality to levels comparable to smoking or obesity ${ }^{4-6}$. However, in the short-term, loneliness is theorized to serve an adaptive, survivalpromoting function, by encouraging social re-connection and self-preservation ${ }^{7}$. This suggests that underlying neural substrates can detect, evaluate, and rectify imbalances in social connection: potentially operating in a homeostatic manner ${ }^{8}$. Uncovering the identity and operation of these neural circuits is key to understanding our fundamental need for social connection and the neurobiological adaptations associated with social deficit 9,10 .

In pursuit of candidate circuitry, we previously proposed that dopamine neurons within the dorsal raphe nucleus marked by the dopamine transporter (DRNDAT) are sensitive to social isolation and can motivate sociability ${ }^{11}$. We showed that acute social isolation potentiates glutamatergic synapses onto DRN ${ }^{D A T}$ neurons and augments their population activity on initial social contact. Strikingly, an analogous endeavor in humans similarly revealed that $10 \mathrm{hr}$ of social isolation heightened midbrain responses to social stimuli ${ }^{12}$. In mice, we further demonstrated that photostimulation of DRNDAT neurons promoted social preference, but also induced place avoidance, suggesting an underlying negative affective state 11. This led us to infer a role for these neurons in motivating social approach, driven by the desire to quell a negative state ${ }^{13}$.

However, the question remains: how do these neurons simultaneously motivate social approach while also inducing a negative affective state? Other studies have shown that, in non-social contexts, DRN ${ }^{\text {DAT }}$ neurons can promote arousal ${ }^{14,15}$, incentive memory expression ${ }^{16}$, antinociception ${ }^{17,18}$, and fear responses ${ }^{19}$. Taken together, this suggests a broad functional role for these neurons in motivating adaptive, survival-promoting behaviors under social and non-social conditions. There are several circuit motifs/neural encoding strategies which could enable this multiplicity of functions: for example distinct functional roles may be associated with projection-defined subpopulations (e.g. ${ }^{20-26}$ ), neurons may simultaneously encode multiple types of information i.e. show 'mixed selectivity' ${ }^{27,28}$, or behavioral output may be governed by context- or state-dependency (e.g. ${ }^{29-32}$ ). Yet, the mechanisms through which DRN ${ }^{D A T}$ neurons exert their influence over social behavior has yet to be unraveled.

Here, we addressed the question of how DRN ${ }^{D A T}$ neurons modulate both sociability and affective state, by exploring the functional role of distinct DRNDAT projections in mice. We show that discrete DRN ${ }^{D A T}$ projections play separable roles in behavior, in spite of their heavily-collateralizing anatomical arrangement. Downstream, we find that within DRN ${ }^{D A T}$ terminal fields, there is spatial segregation of dopamine and neuropeptide receptor expression. Furthermore, photostimulation of DRNDAT inputs can modulate downstream neuronal excitability in a cell-type-specific manner. These findings highlight the anatomical and functional heterogeneity that exists at multiple levels within the DRN ${ }^{\text {AT }}$ system. We suggest this organization may underlie the capacity of the DRNDAT system to exert a broad influence over different forms of behavior: allowing coordinated control over downstream neuronal activity, on distinct timescales, and within disparate target regions. 


\section{RESULTS}

\section{DRN ${ }^{\mathrm{DAT}}$ neurons project to distinct subregions of the amygdala and extended amygdala.}

To explore the circuit motifs ${ }^{33}$ and computational implementation ${ }^{34}$ through which the DRNDAT system might operate, we examined whether discrete DRN ${ }^{D A T}$ projections underlie distinct features of behavior. Prominent DRNDAT projections were identified by quantifying downstream fluorescence following Cre-dependent expression of eYFP in dopamine transporter (DAT)::IRES-Cre mice ${ }^{11,35-37}$ (Fig. S1a-d). We observed distinct patterns of innervation arising from ventral tegmental area (VTA) ${ }^{\mathrm{DAT}}$ and DRNDAT subpopulations (Fig. S1e-h), with DRNDAT projections most densely targeting the oval nucleus of the BNST (ovBNST) and lateral nucleus of the central amygdala $(\mathrm{CeL})$, with weaker, but significant, input to the posterior part of the basolateral amygdala (BLP), consistent with previous tracing studies $16,36,38-40$. Given that the extended amygdala and basolateral amygdala complex have been implicated in aversion- ${ }^{41-44}$ and reward-related processes ${ }^{24,45-47}$, and can elicit autonomic and behavioral changes ${ }^{43}$, we focused on these DRN ${ }^{\text {DAT }}$ projections (Fig. S1h).

\section{Sociability and aversion are mediated by separable DRN ${ }^{\mathrm{DAT}}$ projections.}

We next considered whether DRNDAT projections to the BNST, CeA, and BLP play separable or overlapping roles in modulating behavior. Dopaminergic input to the BNST and CeA has been implicated in threat discrimination ${ }^{48,49}$, anxiety-related behavior ${ }^{50}$, and drug-induced reward ${ }^{51-54}$, while in the BLA complex, dopamine signaling supports both fear ${ }^{55-58}$ and reward learning ${ }^{59,60}$. However, the question remains: which DRN ${ }^{\mathrm{DAT}}$ projections can influence sociability, and which carry valence-related information?

To test the hypothesis that distinct DRNDAT projections promote sociability and induce place avoidance ${ }^{11}$, we performed projection-specific ChR2-mediated photostimulation. We injected an AAV enabling Cre-dependent expression of ChR2 into the DRN of DAT::Cre male mice, and implanted optic fibers over the BNST, CeA, or BLP (Fig. S2a-c). Given that we previously observed that behavioral effects of DRN ${ }^{\text {DAT }}$ photostimulation were predicted by an animal's social rank ${ }^{11}$, we also assessed relative social dominance using the tube test ${ }^{61-63}$ prior to behavioral assays (Fig. 1a and S2d-e).

Firstly, to explore the relationship between social dominance and baseline behavioral profile, we applied a data-driven approach by examining behavioral measures obtained from different assays in a correlation matrix (Fig. S2f). This showed a weak, negative correlation between social dominance and open arm time in the elevated plus maze (EPM) - consistent with a previous report of higher trait anxiety in dominant mice ${ }^{64}$. However, social dominance did not correlate significantly with any other behavioral variable. Furthermore, following dimensionality reduction on baseline behavioral variables, we did not find clearly differentiated clusters of high- and low-ranked mice (Fig. S2g), suggesting that the variation governing these latent features is not related to social rank.

Secondly, to assess how projection-specific photostimulation of DRNDAT terminals affected social preference, we used the three-chamber sociability task ${ }^{65}$, where mice freely-explored a chamber containing a novel juvenile mouse and a novel object at opposite ends (Fig. 1b). This revealed that optical stimulation of the DRNDAT-CeA projection increased social preference (Fig. 1c), but no significant effect was observed with photostimulation of either the DRN ${ }^{D A T}-B N S T$ or DRN ${ }^{D A T}-B L P$ projections. 
113 Furthermore, we found that the optically-induced change in social preference in DRN ${ }^{\text {DAT }}$-CeA mice was 114 positively correlated with social dominance, suggesting that photostimulation elicited a greater increase 115 in sociability in dominant mice (Fig. 1d). This emulates the previous association found with 116 photostimulation at the cell body level ${ }^{11}$.

117 Thirdly, we assessed whether photostimulation was sufficient to support place preference using the real-time place-preference (RTPP) assay. Here, we found that photostimulation of the DRN ${ }^{D A T}$-BLP projection, but not the projection to the BNST or CeA, produced avoidance of the stimulation zone, relative to eYFP controls (Fig. 1e-f). However, we did not find a significant correlation between social dominance and the magnitude of this effect (Fig. $1 \mathrm{~g}$ ). In addition, photostimulation of DRN ${ }^{\text {DAT }}$ projections did not have a detectable effect on locomotion (Fig. S3a-c), anxiety-related behavior (Fig. S3d-f), nor operant responding (Fig. S3g-i).

Fourthly, to gain further insight into the functional divergence of DRNDAT projections in

\section{DRN ${ }^{\mathrm{DAT}}$ neurons show dense collateralization to extended amygdala targets.}

Given the functional separability of distinct DRN ${ }^{\text {DAT }}$ projections, we next considered the anatomical organization of these projections to determine whether form gives rise to function. In other words, we investigated whether DRNDAT outputs exhibit a circuit arrangement that facilitates a coordinated behavioral response. Axonal collateralization is one circuit feature that facilitates coordinated activity across broadly distributed structures ${ }^{71}$. Although VTA DAT projections to striatal and cortical regions typically show little evidence of collateralization ${ }^{72-76}$, in contrast, the DRN serotonergic neurons collateralize heavily to innervate the prefrontal cortex, striatum, midbrain, and amygdala ${ }^{77-79}$. However, it has yet to be determined whether DRNDAT neurons are endowed with this property. 
To assess whether DRNDAT neurons exhibit axon collaterals, we performed dual retrograde tracing with fluorophore-conjugated cholera toxin subunit $B$ (CTB) ${ }^{80}$. We injected each tracer into two of the three downstream sites (BNST, CeA, and/or BLP) (Fig. 2a-b and S4a-c) and, after 7 days for retrograde transport, we examined CTB-expressing cells in the DRN that were co-labelled with tyrosine hydroxylase (TH; Fig. 2C-d). CTB injections into the BNST and CeA resulted in numerous $\mathrm{TH}+$ cells labelled with both CTB-conjugated fluorophores, but significantly fewer dual-labelled cells were observed when injections were placed in the BNST and BLP, or CeA and BLP (Fig. 2d-f). These data suggest significant collateralization to the extended amygdala, which includes the BNST and CeA ${ }^{16,43}$. To confirm the presence of axon collaterals we employed an intersectional viral strategy to selectively label CeA-projecting DRNDAT neurons with cytoplasmic eYFP (Fig. S4d-e). This resulted in eYFPexpressing terminals both in the CeA and in the BNST (Fig. S4f-g).

Taken together, this indicates that DRNDAT axons collateralize heavily to innervate the BNST and $\mathrm{CeA}$, with relatively fewer collaterals innervating the BLP. This anatomical architecture may enable DRNDAT projections to achieve coordinated recruitment of distributed neuronal populations ${ }^{71}$, that may still be locally modulated within the downstream target.

\section{DRN ${ }^{\text {DAT }}$ terminal fields contain spatially-segregated dopamine and neuropeptide receptor populations.}

Our data suggest that DRNDAT projections exert divergent effects over behavior, despite substantial overlap in their upstream cells of origin. Given this overlap, we reasoned that one mechanism through which these projections might achieve distinct behavioral effects is via differential recruitment of downstream signaling pathways. We, therefore, next considered whether the pattern of receptor expression differed within the DRNDAT terminal field of these downstream regions.

Subsets of DRN ${ }^{D A T}$ neurons co-express vasoactive intestinal peptide (VIP) and neuropeptide-W (NPW) ${ }^{81-83}$, and so we examined both dopamine (Drd1 and Drd2) and neuropeptide (Vipr2 and Npbwr1) receptor expression within DRNDAT terminal fields. To achieve this, we performed single molecule fluorescence in situ hybridization (smFISH) using RNAscope (Fig. S5a-b). In the BNST and CeA we observed a strikingly similar pattern of receptor expression with dense neuropeptide receptor expression in the oval BNST and ventromedial CeL, and a high degree of co-localization (Fig. 3a-h and S5c-h). In the BNST and CeA subregions containing the highest density of DRNDAT terminals, dopamine receptor expression was relatively more sparse, with $\operatorname{Drd} 2$ more abundant than $\operatorname{Drd} 1$, as previously described $47,48,50,84,85$ (Fig. 3a-h). The DRNDAT terminal field of the BLP displayed a markedly different receptor expression pattern, dominated by Drd1 (Fig. 3i-I and S5i-k), consistent with previous reports 50,59,84. Thus, in contrast to the BNST and CeA, the effects of DRNDAT input to the BLP may be predominantly mediated via $D_{1}$-receptor signaling. Collectively, this expression pattern suggests that the dopamine- and neuropeptide-mediated effects of DRNDAT input may be spatially-segregated within downstream regions - providing the infrastructure for divergent modulation of cellular subsets. 
DRN ${ }^{\text {DAT }}$ input has divergent effects on downstream cellular excitability.

Our data suggest that DRN DAT projections exert divergent effects over behavior, despite substantial collateralization. One mechanism through which these projections might achieve distinct behavioral effects is via differential modulation of activity in downstream neurons. The multi-transmitter phenotype of DRNDAT neurons ${ }^{81,82,86,87}$, regionally-distinct downstream receptor expression, and the observed pre- and post-synaptic actions of exogenously applied dopamine ${ }^{88-95}$ provides optimal conditions for diverse modulation of neural activity. However, it remains unknown how temporally precise activation of DRNDAT terminals influences excitability at the single-cell level.

We, therefore, next examined how DRNDAT input affects downstream excitability. To achieve this, we expressed ChR2 in DRN ${ }^{\text {DAT }}$ neurons, and used ex vivo electrophysiology to record from downstream neurons (Fig. 4a-c and S6a-c). Optical stimulation at the resting membrane potential evoked both excitatory and inhibitory post-synaptic potentials (EPSPs and IPSPs) in downstream cells (Fig. 4d-f), which were typically monosynaptic (Fig. S6d-e). During spontaneous firing, BNST cells were excited and BLP cells mostly inhibited by DRN ${ }^{D A T}$ optical stimulation, whereas a mixture of responses was observed in the CeA (Fig. 4g-k and S6f-g). The fast rise and decay kinetics of the EPSP suggest an AMPAR-mediated potential, resulting from glutamate co-release ${ }^{11,17}$, whereas the slow IPSP kinetics are consistent with opening of GIRK channels, which can occur via $D_{2}$-receptor ${ }^{96,97}$ or GABAB receptor signaling ${ }^{98-100}$.

Given the diversity of responses observed in the CeA and BLP, we next examined these downstream cells in more detail. To assess whether baseline electrophysiological properties predicted the optically-evoked response, we used unsupervised agglomerative hierarchical clustering to classify downstream cells (Fig. 4l-m). This established approach has been successfully applied to electrophysiological datasets to reveal distinct neuronal subclasses ${ }^{101-103}$. The resulting dendrograms yielded two major clusters in the CeA and BLP, with distinct electrophysiological characteristics (Fig. $4 \mathrm{n}-\mathrm{q}$ and S6h-k). CeA cells in cluster 1 represented 'late-firing' neurons, whereas cluster 2 were typical of 'regular-firing' neurons ${ }^{104-106}$. Strikingly, these clusters exhibited dramatically different responses to DRNDAT photostimulation, with cluster 1 'late-firing' neurons excited and cluster 2 'regular-firing' neurons mostly inhibited (Fig. 4o). Similarly, BLP cells delineated into two major clusters, with properties characteristic of pyramidal neurons (cluster 1) and GABAergic interneurons (cluster 2) (Fig. 4p-q). These clusters showed remarkably different responses to DRNDAT input, with $93 \%$ of putative pyramidal neurons showing an inhibitory response, and $62 \%$ of putative GABAergic interneurons showing an excitatory response (Fig. 4q). In addition, clustering $\mathrm{CeA}$ and BLP cells together yielded a very similar result (Fig. S6I-n), perhaps reflecting similar anatomical architecture that can be repurposed towards distinct functions. Thus, while photoactivation of DRNDAT terminals elicits heterogeneous responses in downstream neurons, baseline cell properties strongly predict their response, suggesting robust synaptic organization. The opposing nature of these responses, in different neuronal subsets, suggests that - rather than inducing an overall augmentation or suppression of activity - DRNDAT input may adjust the pattern of downstream activity, in order to exert a functional shift in behavior.

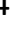


Neural circuits which motivate social approach are essential in maintaining social connections and preventing isolation. Here we show that DRN ${ }^{D A T}$ neurons can exert a multi-faceted influence over behavior, with the pro-social effects mediated by the projection to the CeA. Our data suggest these effects are enabled via separable functional projections, dense collateralization, co-transmission, and precisely organized synaptic connectivity. Taken together, these circuit features may facilitate a coordinated, but flexible, response in the presence of social stimuli, that can be flexibly guided based on internal social homeostatic need state.

\section{DRN ${ }^{\text {DAT }}$ circuit arrangement enables a broadly distributed, coordinated response.}

Our findings revealed several features of the DRNDAT circuit which might facilitate a concerted response to novel social and non-social situations. Firstly, we observed dissociable roles for discrete downstream projections - a common motif of valence-encoding neural circuits ${ }^{33}$. Biased recruitment of these 'divergent paths' 33 to the BNST, CeA, and BLP by upstream inputs may serve to fine-tune the balance between social investigation and environmental exploration: facilitating behavioral flexibility with changing environmental conditions or internal state. Secondly, we demonstrate extensive collateralization of DRN ${ }^{\text {DAT }}$ neurons. In other populations, collateralization is proposed to aid temporal coordination of a multifaceted response: enabling synchronous activation of distributed regions ${ }^{71,79}$. This feature may, therefore, facilitate coordinated recruitment of the BNST and CeA, allowing these regions to work in concert to promote social approach while also maintaining vigilance to salient environmental stimuli. Thirdly we find precise synaptic organization in the DRNDAT modulation of downstream neuronal activity. Combined with the spatially-segregated downstream receptor expression pattern, this organization may allow DRNDAT neurons to elicit broad, yet finely-tuned, control over the pattern of neuronal activity, on multiple timescales.

\section{Separable projections mediate social behavior and valence.}

Our data supports the hypothesis that separable DRNDAT projections mediate distinct functional roles: a feature which has been previously observed in other neuronal circuits (e.g. $\left.{ }^{20-24}\right)$. The DRNDAT circuit attributes we describe above may further enable this system to modulate other diverse forms of behavior (e.g. arousal 14, fear/reward associations 16,19, and antinociception ${ }^{17,18}$ ). These could be mediated via other downstream projections and/or via these same projections under different environmental contexts, testing conditions, and/or internal states. Further work is required to determine how this system is able to exert a broad influence over multiple forms of behavior. Collectively, however, our data and others support a role for the DRN ${ }^{\mathrm{DAT}}$ system in exerting a coordinated behavioral response to novel situations - both social and non-social.

The CeA has been implicated in mediating the response to threats - orchestrating defensive behavioral responses and autonomic changes via efferents to subcortical and brainstem nuclei ${ }^{41,107-}$

109. One possible interpretation, therefore, is that DRNDAT input to the CeA suppresses fear-promoting neuronal ensembles in order to facilitate social approach. In the maintenance of social homeostasis, suppression of fear in the presence of social stimuli may represent an adaptive response - preventing salient social stimuli from being interpreted as a threat. Indeed, other need states, such as hunger, are associated with fear suppression and higher-risk behavior ${ }^{110}$, suggesting a conserved response to 
homeostatic imbalance ${ }^{8}$. However, the motivation to attend to social stimuli may also be driven by territorial defense, highlighting a need to further understand how internal states can play into the output of this system. A more comprehensive knowledge of the functional cell-types modulated by DRNDAT activity will facilitate our understanding of how this input can shape the downstream neuronal representation of social and non-social stimuli.

In contrast to the CeA, photoactivation of the DRNDAT-BLP projection produced avoidance of the stimulation zone, suggesting an aversive state. This differs from the valence-independent role of VTA dopamine input to the greater BLA complex, wherein dopamine signaling gates synaptic plasticity for associative learning of both positive and negative valence ${ }^{60}$ and responds to salient stimuli predicting both positive and negative outcomes ${ }^{59}$. However, DRN and VTA axonal fields differ within the BLA complex, with DRN ${ }^{D A T}$ terminals being more concentrated within the BLP, and VTA ${ }^{D A T}$ inputs traversing the LA, BLA and intercalated cells more densely.

While there have been seemingly contradictory reports on the effect of dopamine on excitability in the BLA, our observations using photostimulation of DRN ${ }^{D A T}$ terminals (in short phasic bursts) are consistent with in vivo extracellular recordings combined with electrical stimulation of the midbrain ${ }^{93}$. One unifying hypothesis is that dopamine induces an indirect GABA-mediated suppression of pyramidal neurons, which may attenuate their response to weak inputs, while directly exciting pyramidal neurons to augment their response to large inputs ${ }^{90,93}$. In this way, amygdala dopamine may underlie a similar role to cortical dopamine ${ }^{111}$ : enhancing signal-to-noise ratio to facilitate behavioral responses to salient stimuli ${ }^{112}$.

\section{Multi-transmitter phenotype of DRN ${ }^{\mathrm{DAT}}$ neurons may permit modulation on different timescales.}

DRN ${ }^{\text {DAT }}$ neurons possess an impressive repertoire of signaling molecules: alongside dopamine and glutamate subsets of DRN ${ }^{\text {DAT }}$ neuron express VIP and NPW ${ }^{81-83}$. While there is some partial segregation of VIP- and NPW-expressing neurons ${ }^{82}$, our receptor expression analyses suggest that these neuropeptides converge on the same neurons in the BNST and CeA. This co-localization is intriguing, given that Vipr2 is typically coupled to the excitatory $\mathrm{G}_{\mathrm{s}}$-protein ${ }^{113}$, while $\mathrm{Npbwr} 1$ is coupled to the inhibitory $\mathrm{G}_{\text {i-protein }}{ }^{114,115}$. Therefore, signaling through these receptors may exert opposing actions on downstream cells. Recruitment of neuropeptidergic signaling pathways may support slower, sustained downstream modulation, for example, in hunger-mediating hypothalamic Agouti-Related Peptide (AgRP) neurons, neuropeptide co-release is essential for sustaining feeding behavior ${ }^{116}$. Therefore, a delayed, persistent neuropeptide-mediated signal might enable downstream modulation to outlive phasic DRNDAT activity: promoting behavioral adjustments over longer timescales.

While the functional role of these neuropeptides remains to be elucidated, studies on knockout mice suggest a role for NPW in social behavior and stress responding ${ }^{114}$. ${ }^{83}$. Furthermore, humans with a single-nucleotide polymorphism (SNP) of the NPBWR1 gene (which impairs receptor function) perceive fearful/angry faces as more positive and less submissive ${ }^{117}$, indicating a role for NPW signaling in interpretating social signals. Similarly, the function of DRN VIP+ neurons has received little attention in rodent models, but there has been more focus on the role of VIP in avian social behavior 118. Of particular interest, in the rostral arcopallium (a homolog of mammalian amygdala ${ }^{119}$ ), VIP binding density is elevated in birds during seasonal flocking ${ }^{120}$. This suggests that elevated VIP receptor expression may encourage affiliative social grouping behavior in birds ${ }^{120}$. Thus, NPW and VIP may act 
320 in concert with fast glutamate-mediated and slow dopamine-mediated neurotransmission in the central 321 extended amygdala, to modulate behavior on different timescales.

322

\section{Conclusion}

324 Our findings support a role for DRNDAT projections in promoting distinct features of the response 325 to novel social stimuli - orchestrating a coordinated, flexible response through recruitment of specific 326 downstream circuits. This highlights the breadth of DRNDAT influence over downstream targets, the 327 signaling complexity of this system, and its potential to underlie a shift in both behavior and affective 328 state. Uncovering the neural circuit mechanisms which incline individuals towards sociability is key to 329 understanding the basic human need for social connection and the neural representation of loneliness. 


\section{ACKNOWLEDGEMENTS}

K.M.T. is the Wylie Vale Chair at the Salk Institute for Biological Studies, a New York Stem Cell Foundation - Robertson Investigator, and a McKnight Scholar. This work was supported by funding from the JPB Foundation, Alfred P Sloan Foundation, New York Stem Cell Foundation, Klingenstein Foundation, McKnight Foundation, Clayton Foundation, Dolby Family Fund, R01-MH115920 (NIMH), the NIH Director's New Innovator Award DP2-DK102256 (NIDDK), and Pioneer Award DP1-AT009925 (NCCIH). G.A.M was supported by a Postdoctoral Research Fellowship from the Charles A. King Trust. R.L.M. was funded through the MSRP program in the Brains \& Cognitive Sciences Department at MIT, supported by the Center for Brains, Minds and Machines (CBMM), and funded by NSF STC award CCF-1231216. E.M.B was supported by a summer scholarship from Johnson \& Johnson. We thank C. Leppla, J. Olsen, P. Namburi, V. Barth, J. Wang, K. Batra, A. Brown, and A. Libster for technical advice, all members of the Tye Lab for helpful discussion, and advice from the CellProfiler team at the Broad Institute. We also thank Rachel Neve for the HSV construct, and Charu Ramakrishnan \& Karl Deisseroth for $\mathrm{AAV}_{5}$-fDIO-eYFP.

\section{AUTHOR CONTRIBUTIONS}

K.M.T. and G.A.M. conceptualized the project, designed experiments, supervised experiments, and directed data analyses. M.E.L and M.B. performed and analyzed smFISH experiments. G.A.M., M.E.L., C.R.L., E.M.B., E.P., G.S.P., A.L.M., and A.P.L. performed stereotaxic surgeries. G.A.M., E.M.B, E.P, G.S.P, M.E.L., M.B., A.L.M, R.M., C.R.L., and A.P.L. ran optogenetic manipulation experiments and analyzed behavioral data. L.K. performed Markov model analysis. G.A.M., E.M.B., E.P., G.S.P., A.L.M., and A.P.L. performed immunohistochemistry and analyzed images. G.A.M performed ex vivo electrophysiology, and N.P.C., E.Y.K, and R.W. contributed to experimental design and data interpretation. G.A.M. and K.M.T. wrote the manuscript with review and editing from M.E.L., C.R.L., E.M.B., M.B., R.M., L.K., E.P., G.S.P., A.L.M., A.P.L., E.Y.K., N.P-C., and R.W. 


\section{REFERENCES}

359 1. Koto, A., Mersch, D., Hollis, B. \& Keller, L. Social isolation causes mortality by disrupting energy

360 homeostasis in ants. Behav. Ecol. Sociobiol. 69, 583-591 (2015).

361 2. Silk, J. B. et al. Strong and Consistent Social Bonds Enhance the Longevity of Female Baboons. Curr. Biol.

$362 \quad 20,1359-1361(2010)$.

363 3. Yee, J. R., Cavigelli, S. A., Delgado, B. \& McClintock, M. K. Reciprocal affiliation among adolescent rats 364 during a mild group stressor predicts mammary tumors and lifespan. Psychosom. Med. 70, 1050-1059 365 (2008).

366 4. Holt-Lunstad, J., Smith, T. B. \& Layton, J. B. Social Relationships and Mortality Risk: A Meta-analytic 367 Review. PLoS Med 7, e1000316 (2010).

368 5. Holt-Lunstad, J., Smith, T. B., Baker, M., Harris, T. \& Stephenson, D. Loneliness and social isolation as risk 369 factors for mortality: a meta-analytic review. Perspect. Psychol. Sci. J. Assoc. Psychol. Sci. 10, 227-237 (2015).

6. Pantell, M. et al. Social Isolation: A Predictor of Mortality Comparable to Traditional Clinical Risk Factors. Am. J. Public Health 103, 2056-2062 (2013).

7. Cacioppo, J. T. \& Cacioppo, S. Chapter Three - Loneliness in the Modern Age: An Evolutionary Theory of Loneliness (ETL). in Advances in Experimental Social Psychology (ed. Olson, J. M.) vol. 58 127-197 (Academic Press, 2018).

8. Matthews, G. A. \& Tye, K. M. Neural mechanisms of social homeostasis. Ann. N. Y. Acad. Sci. 1457, 5-25 (2019).

9. Lee, C. R., Chen, A. \& Tye, K. M. The neural circuitry of social homeostasis: Consequences of acute versus chronic social isolation. Cell 184, 1500-1516 (2021).

10. Tomova, L., Tye, K. \& Saxe, R. The neuroscience of unmet social needs. Soc. Neurosci. 0, 1-11 (2019).

11. Matthews, G. A. et al. Dorsal Raphe Dopamine Neurons Represent the Experience of Social Isolation. Cell 164, 617-631 (2016). 
12. Tomova, L. et al. Acute social isolation evokes midbrain craving responses similar to hunger. Nat. Neurosci. 23, 1597-1605 (2020).

13. Hull, C. L. Principles of Behavior: An Introduction to Behavior Theory. (D. Appleton-Century Company, Incorporated, 1943).

14. Cho, J. R. et al. Dorsal Raphe Dopamine Neurons Modulate Arousal and Promote Wakefulness by Salient Stimuli. Neuron 94, 1205-1219.e8 (2017).

16. Lin, R. et al. The Raphe Dopamine System Controls the Expression of Incentive Memory. Neuron 106,

15. Lu, J., Jhou, T. C. \& Saper, C. B. Identification of wake-active dopaminergic neurons in the ventral periaqueductal gray matter. J. Neurosci. Off. J. Soc. Neurosci. 26, 193-202 (2006). 498-514.e8 (2020).

17. Li, C. et al. Mu Opioid Receptor Modulation of Dopamine Neurons in the Periaqueductal Gray/Dorsal Raphe: A Role in Regulation of Pain. Neuropsychopharmacol. Off. Publ. Am. Coll. Neuropsychopharmacol. 41, 2122-2132 (2016).

18. Meyer, P. J., Morgan, M. M., Kozell, L. B. \& Ingram, S. L. Contribution of dopamine receptors to periaqueductal gray-mediated antinociception. Psychopharmacology (Berl.) 204, 531-540 (2009).

19. Groessl, F. et al. Dorsal tegmental dopamine neurons gate associative learning of fear. Nat. Neurosci. 21, 952-962 (2018).

20. Han, W. et al. Integrated Control of Predatory Hunting by the Central Nucleus of the Amygdala. Cell 168, 311-324.e18 (2017).

21. Kim, S.-Y. et al. Diverging neural pathways assemble a behavioural state from separable features in anxiety. Nature 496, 219-223 (2013).

22. Kohl, J. et al. Functional circuit architecture underlying parental behaviour. Nature 556, 326-331 (2018).

23. Lammel, S., Ion, D. I., Roeper, J. \& Malenka, R. C. Projection-specific modulation of dopamine neuron synapses by aversive and rewarding stimuli. Neuron 70, 855-862 (2011). 
407

408

409

410

411

412

413

414

415

416

417

24. Namburi, P. et al. A circuit mechanism for differentiating positive and negative associations. Nature 520, 675-678 (2015).

25. Senn, V. et al. Long-Range Connectivity Defines Behavioral Specificity of Amygdala Neurons. Neuron 81, 428-437 (2014).

26. Tye, K. M. et al. Amygdala circuitry mediating reversible and bidirectional control of anxiety. Nature 471, 358-362 (2011).

27. Rigotti, M. et al. The importance of mixed selectivity in complex cognitive tasks. Nature $497,585-590$ (2013).

28. Tian, J. et al. Distributed and Mixed Information in Monosynaptic Inputs to Dopamine Neurons. Neuron 91, 1374-1389 (2016).

29. Krzywkowski, P., Penna, B. \& Gross, C. T. Dynamic encoding of social threat and spatial context in the hypothalamus. eLife 9 , e57148 (2020).

30. Kyriazi, P., Headley, D. B. \& Pare, D. Multi-dimensional Coding by Basolateral Amygdala Neurons. Neuron 99, 1315-1328.e5 (2018).

31. Lemos, J. C. et al. Severe stress switches CRF action in the nucleus accumbens from appetitive to aversive. Nature 490, 402-406 (2012).

32. Seo, C. et al. Intense Threat Switches Dorsal Raphe Serotonin Neurons to a Paradoxical Operational Mode. Science 363, 538-542 (2019).

33. Tye, K. M. Neural Circuit Motifs in Valence Processing. Neuron 100, 436-452 (2018).

34. Lockwood, P. L., Apps, M. A. J. \& Chang, S. W. C. Is There a 'Social' Brain? Implementations and Algorithms. Trends Cogn. Sci. 24, 802-813 (2020).

35. Bäckman, C. M. et al. Characterization of a mouse strain expressing Cre recombinase from the 3' untranslated region of the dopamine transporter locus. Genes. N. Y. N 2000 44, 383-390 (2006).

36. Cardozo Pinto, D. F. et al. Characterization of transgenic mouse models targeting neuromodulatory systems reveals organizational principles of the dorsal raphe. Nat. Commun. 10, 4633 (2019). 
432

433

434

435

436

437

438

439

440

441

442

443

444

445

446

447

448

449

450

451

452

453

454

455

37. Lammel, S. et al. Diversity of transgenic mouse models for selective targeting of midbrain dopamine neurons. Neuron 85, 429-438 (2015).

38. Hasue, R. H. \& Shammah-Lagnado, S. J. Origin of the dopaminergic innervation of the central extended amygdala and accumbens shell: a combined retrograde tracing and immunohistochemical study in the rat. J. Comp. Neurol. 454, 15-33 (2002).

39. Meloni, E. G., Gerety, L. P., Knoll, A. T., Cohen, B. M. \& Carlezon, W. A. Behavioral and Anatomical Interactions between Dopamine and Corticotropin-Releasing Factor in the Rat. J. Neurosci. 26, 38553863 (2006).

40. Oh, S. W. et al. A mesoscale connectome of the mouse brain. Nature 508, 207-214 (2014).

41. Davis, M., Walker, D. L., Miles, L. \& Grillon, C. Phasic vs Sustained Fear in Rats and Humans: Role of the Extended Amygdala in Fear vs Anxiety. Neuropsychopharmacology 35, 105-135 (2010).

42. Goode, T. D. \& Maren, S. Role of the bed nucleus of the stria terminalis in aversive learning and memory. Learn. Mem. 24, 480-491 (2017).

43. Janak, P. H. \& Tye, K. M. From circuits to behaviour in the amygdala. Nature 517, 284-292 (2015).

44. Lebow, M. A. \& Chen, A. Overshadowed by the amygdala: the bed nucleus of the stria terminalis emerges as key to psychiatric disorders. Mol. Psychiatry 21, 450-463 (2016).

45. Douglass, A. M. et al. Central amygdala circuits modulate food consumption through a positive-valence mechanism. Nat. Neurosci. 20, 1384-1394 (2017).

46. Jennings, J. H. et al. Distinct extended amygdala circuits for divergent motivational states. Nature 496, 224-228 (2013).

47. Kim, J., Zhang, X., Muralidhar, S., LeBlanc, S. A. \& Tonegawa, S. Basolateral to Central Amygdala Neural Circuits for Appetitive Behaviors. Neuron 93, 1464-1479.e5 (2017).

48. De Bundel, D. et al. Dopamine D2 receptors gate generalization of conditioned threat responses through mTORC1 signaling in the extended amygdala. Mol. Psychiatry 21, 1545-1553 (2016). 
49. Jo, Y. S., Heymann, G. \& Zweifel, L. S. Dopamine Neurons Reflect the Uncertainty in Fear Generalization. Neuron 100, 916-925.e3 (2018).

50. Perez de la Mora, M. et al. Distribution of dopamine $\mathrm{D}(2)$-like receptors in the rat amygdala and their role in the modulation of unconditioned fear and anxiety. Neuroscience 201, 252-266 (2012).

51. Eiler, W. J. A., Seyoum, R., Foster, K. L., Mailey, C. \& June, H. L. D1 dopamine receptor regulates alcoholmotivated behaviors in the bed nucleus of the stria terminalis in alcohol-preferring (P) rats. Synap. N. Y. N 48, 45-56 (2003).

52. Epping-Jordan, M. P., Markou, A. \& Koob, G. F. The dopamine D-1 receptor antagonist SCH 23390 injected into the dorsolateral bed nucleus of the stria terminalis decreased cocaine reinforcement in the rat. Brain Res. 784, 105-115 (1998).

53. Rezayof, A., Zarrindast, M.-R., Sahraei, H. \& Haeri-Rohani, A.-H.-R. Involvement of dopamine D2 receptors of the central amygdala on the acquisition and expression of morphine-induced place preference in rat. Pharmacol. Biochem. Behav. 74, 187-197 (2002).

54. Thiel, K. J. et al. Stimulation of dopamine D2/D3 but not D1 receptors in the central amygdala decreases cocaine-seeking behavior. Behav. Brain Res. 214, 386-394 (2010).

55. Bissière, S., Humeau, Y. \& Luthi, A. Dopamine gates LTP induction in lateral amygdala by suppressing feedforward inhibition. Nat. Neurosci. 6, 587-592 (2003).

56. Fadok, J. P., Dickerson, T. M. K. \& Palmiter, R. D. Dopamine Is Necessary for Cue-Dependent Fear Conditioning. J. Neurosci. 29, 11089-11097 (2009).

57. Guarraci, F. A., Frohardt, R. J. \& Kapp, B. S. Amygdaloid D1 dopamine receptor involvement in Pavlovian fear conditioning. Brain Res. 827, 28-40 (1999).

58. de Oliveira, A. R. et al. Conditioned fear is modulated by D2 receptor pathway connecting the ventral tegmental area and basolateral amygdala. Neurobiol. Learn. Mem. 95, 37-45 (2011).

59. Lutas, A. et al. State-specific gating of salient cues by midbrain dopaminergic input to basal amygdala. Nat. Neurosci. 22, 1820-1833 (2019). 
481

482

483

484

485

486

487

488

489

490

491

492

493

494

495

496

497

498

499

500

501

502

503

60. Tye, K. M. et al. Methylphenidate facilitates learning-induced amygdala plasticity. Nat. Neurosci. 13, 475481 (2010).

61. Lindzey, G., Winston, H. \& Manosevitz, M. Social Dominance in Inbred Mouse Strains. Nature 191, 474476 (1961).

62. Wang, F. et al. Bidirectional Control of Social Hierarchy by Synaptic Efficacy in Medial Prefrontal Cortex. Science 334, 693-697 (2011).

63. Zhou, T., Sandi, C. \& Hu, H. Advances in understanding neural mechanisms of social dominance. Curr. Opin. Neurobiol. 49, 99-107 (2018).

64. Larrieu, T. et al. Hierarchical Status Predicts Behavioral Vulnerability and Nucleus Accumbens Metabolic Profile Following Chronic Social Defeat Stress. Curr. Biol. 27, 2202-2210.e4 (2017).

65. Moy, S. S. et al. Sociability and preference for social novelty in five inbred strains: an approach to assess autistic-like behavior in mice. Genes Brain Behav. 3, 287-302 (2004).

66. Bailey, K. R. \& Crawley, J. N. Anxiety-Related Behaviors in Mice. in Methods of Behavior Analysis in Neuroscience (ed. Buccafusco, J. J.) (CRC Press/Taylor \& Francis, 2009).

67. Lever, C., Burton, S. \& O'Keefe, J. Rearing on hind legs, environmental novelty, and the hippocampal formation. Rev. Neurosci. 17, 111-133 (2006).

68. Füzesi, T., Daviu, N., Wamsteeker Cusulin, J. I., Bonin, R. P. \& Bains, J. S. Hypothalamic CRH neurons orchestrate complex behaviours after stress. Nat. Commun. 7, 11937 (2016).

69. Lee, W., Fu, J., Bouwman, N., Farago, P. \& Curley, J. P. Temporal microstructure of dyadic social behavior during relationship formation in mice. PLOS ONE 14, (2019).

70. Tejada, J., Bosco, G. G., Morato, S. \& Roque, A. C. Characterization of the rat exploratory behavior in the elevated plus-maze with Markov chains. J. Neurosci. Methods 193, 288-295 (2010).

71. Rockland, K. S. Axon Collaterals and Brain States. Front. Syst. Neurosci. 12, (2018). 
504 72. Aransay, A., Rodríguez-López, C., García-Amado, M., Clascá, F. \& Prensa, L. Long-range projection 505 neurons of the mouse ventral tegmental area: a single-cell axon tracing analysis. Front. Neuroanat. 9, $506 \quad$ (2015).

507 73. Beier, K. T. et al. Circuit Architecture of VTA Dopamine Neurons Revealed by Systematic Input-Output $508 \quad$ Mapping. Cell 162, 622-634 (2015).

509 74. Lerner, T. N. et al. Intact-Brain Analyses Reveal Distinct Information Carried by SNc Dopamine Subcircuits. $510 \quad$ Cell 162, 635-647 (2015).

511 75. Matsuda, W. et al. Single Nigrostriatal Dopaminergic Neurons Form Widely Spread and Highly Dense 512 Axonal Arborizations in the Neostriatum. J. Neurosci. 29, 444-453 (2009).

513 76. Moore, R. Y. \& Bloom, F. E. Central catecholamine neuron systems: anatomy and physiology of the 514 dopamine systems. Annu. Rev. Neurosci. 1, 129-169 (1978).

515 77. Gagnon, D. \& Parent, M. Distribution of VGLUT3 in Highly Collateralized Axons from the Rat Dorsal Raphe $516 \quad$ Nucleus as Revealed by Single-Neuron Reconstructions. PLOS ONE 9, e87709 (2014).

517 78. van der Kooy, D. \& Hattori, T. Dorsal raphe cells with collateral projections to the caudate-putamen and 518 substantia nigra: a fluorescent retrograde double labeling study in the rat. Brain Res. 186, 1-7 (1980).

519 79. Waselus, M., Valentino, R. J. \& Van Bockstaele, E. J. Collateralized dorsal raphe nucleus projections: a mechanism for the integration of diverse functions during stress. J. Chem. Neuroanat. 41, 266-280 (2011).

80. Beyeler, A. et al. Organization of Valence-Encoding and Projection-Defined Neurons in the Basolateral Amygdala. Cell Rep. 22, 905-918 (2018).

81. Dougalis, A. G. et al. Functional properties of dopamine neurons and co-expression of vasoactive intestinal polypeptide in the dorsal raphe nucleus and ventro-lateral periaqueductal grey. Eur. J. Neurosci. 36, 3322-3332 (2012). (2019). 
529 83. Motoike, T. et al. Mesolimbic neuropeptide W coordinates stress responses under novel environments.

$530 \quad$ Proc. Natl. Acad. Sci. U. S. A. 113, 6023-6028 (2016).

531 84. McCullough, K. M., Daskalakis, N. P., Gafford, G., Morrison, F. G. \& Ressler, K. J. Cell-type-specific interrogation of CeA Drd2 neurons to identify targets for pharmacological modulation of fear extinction. Transl. Psychiatry 8, (2018).

85. McCullough, K. M., Morrison, F. G., Hartmann, J., Carlezon, W. A. \& Ressler, K. J. Quantified Coexpression Analysis of Central Amygdala Subpopulations. eNeuro 5, (2018).

86. Dougalis, A. G., Matthews, G. A. C., Liss, B. \& Ungless, M. A. lonic currents influencing spontaneous firing and pacemaker frequency in dopamine neurons of the ventrolateral periaqueductal gray and dorsal raphe nucleus (vIPAG/DRN): A voltage-clamp and computational modelling study. J. Comput. Neurosci. 42, 275-305 (2017).

87. Poulin, J.-F. et al. Mapping projections of molecularly defined dopamine neuron subtypes using intersectional genetic approaches. Nat. Neurosci. 21, 1260-1271 (2018).

88. Kash, T. L., Nobis, W. P., Matthews, R. T. \& Winder, D. G. Dopamine Enhances Fast Excitatory Synaptic Transmission in the Extended Amygdala by a CRF-R1-Dependent Process. J. Neurosci. 28, 13856-13865 (2008).

89. Krawczyk, M. et al. Double-Dissociation of the Catecholaminergic Modulation of Synaptic Transmission in the Oval Bed Nucleus of the Stria Terminalis. J. Neurophysiol. 105, 145-153 (2010).

90. Kröner, S., Rosenkranz, J. A., Grace, A. A. \& Barrionuevo, G. Dopamine Modulates Excitability of Basolateral Amygdala Neurons In Vitro. J. Neurophysiol. 93, 1598-1610 (2005).

91. Marowsky, A., Yanagawa, Y., Obata, K. \& Vogt, K. E. A Specialized Subclass of Interneurons Mediates Dopaminergic Facilitation of Amygdala Function. Neuron 48, 1025-1037 (2005).

92. Naylor, J. C. et al. Dopamine attenuates evoked inhibitory synaptic currents in central amygdala neurons. Eur. J. Neurosci. 32, 1836-1842 (2010). 
553

554

555

556

557

558

559

560

561

562

563

564

565

566

567

568

569

570

571

572

573

574

575

576

93. Rosenkranz, J. A. \& Grace, A. A. Modulation of basolateral amygdala neuronal firing and afferent drive by dopamine receptor activation in vivo. J. Neurosci. Off. J. Soc. Neurosci. 19, 11027-11039 (1999).

94. Rosenkranz, J. A. \& Grace, A. A. Cellular mechanisms of infralimbic and prelimbic prefrontal cortical inhibition and dopaminergic modulation of basolateral amygdala neurons in vivo. J. Neurosci. Off. J. Soc. Neurosci. 22, 324-337 (2002).

95. Silberman, Y. \& Winder, D. G. Corticotropin releasing factor and catecholamines enhance glutamatergic neurotransmission in the lateral subdivision of the central amygdala. Neuropharmacology 70, 316-323 (2013).

96. Beckstead, M. J., Grandy, D. K., Wickman, K. \& Williams, J. T. Vesicular dopamine release elicits an inhibitory postsynaptic current in midbrain dopamine neurons. Neuron 42, 939-946 (2004).

97. Marcott, P. F. et al. Regional heterogeneity of D2-receptor signaling in the dorsal striatum and nucleus accumbens. Neuron 98, 575-587.e4 (2018).

98. Bettler, B., Kaupmann, K., Mosbacher, J. \& Gassmann, M. Molecular structure and physiological functions of GABA(B) receptors. Physiol. Rev. 84, 835-867 (2004).

99. Destexhe, A. \& Sejnowski, T. J. G protein activation kinetics and spillover of gamma-aminobutyric acid may account for differences between inhibitory responses in the hippocampus and thalamus. Proc. Natl. Acad. Sci. U. S. A. 92, 9515-9519 (1995).

100. Mackay, J. P. et al. NPY2 Receptors Reduce Tonic Action Potential-Independent GABAB Currents in the Basolateral Amygdala. J. Neurosci. 39, 4909-4930 (2019).

101. Cauli, B. et al. Classification of fusiform neocortical interneurons based on unsupervised clustering. Proc. Natl. Acad. Sci. U. S. A. 97, 6144-6149 (2000).

102. Guthman, E. M. et al. Cell-type-specific control of basolateral amygdala neuronal circuits via entorhinal cortex-driven feedforward inhibition. eLife https://elifesciences.org/articles/50601/figures (2020) doi:10.7554/eLife.50601. 
577

578

579

580

581

582

583

584

585

586

587

588

589

590

591

592

593

594

595

596

597

598

599

600

103. Hou, W.-H. et al. Wiring Specificity and Synaptic Diversity in the Mouse Lateral Central Amygdala. J. Neurosci. Off. J. Soc. Neurosci. 36, 4549-4563 (2016).

104. Chieng, B. C. H., Christie, M. J. \& Osborne, P. B. Characterization of neurons in the rat central nucleus of the amygdala: cellular physiology, morphology, and opioid sensitivity. J. Comp. Neurol. 497, 910-927 (2006).

105. Dumont, E. C., Martina, M., Samson, R. D., Drolet, G. \& Paré, D. Physiological properties of central amygdala neurons: species differences. Eur. J. Neurosci. 15, 545-552 (2002).

106. Lopez de Armentia, M. \& Sah, P. Firing properties and connectivity of neurons in the rat lateral central nucleus of the amygdala. J. Neurophysiol. 92, 1285-1294 (2004).

107. Fadok, J. P., Markovic, M., Tovote, P. \& Lüthi, A. New perspectives on central amygdala function. Curr. Opin. Neurobiol. 49, 141-147 (2018).

108. Gungor, N. Z. \& Paré, D. Functional Heterogeneity in the Bed Nucleus of the Stria Terminalis. J. Neurosci. 36, 8038-8049 (2016).

109. Tovote, P. et al. Midbrain circuits for defensive behaviour. Nature 534, 206-212 (2016).

110. Padilla, S. L. et al. AgRP Neural Circuits Mediate Adaptive Behaviors in the Starved State. Nat. Neurosci. 19, 734-741 (2016).

111. Gulledge, A. T. \& Jaffe, D. B. Multiple effects of dopamine on layer V pyramidal cell excitability in rat prefrontal cortex. J. Neurophysiol. 86, 586-595 (2001).

112. Vander Weele, C. M. et al. Dopamine enhances signal-to-noise ratio in cortical-brainstem encoding of aversive stimuli. Nature 563, 397 (2018).

113. White, C. M., Ji, S., Cai, H., Maudsley, S. \& Martin, B. Therapeutic potential of vasoactive intestinal peptide and its receptors in neurological disorders. CNS Neurol. Disord. Drug Targets 9, 661 (2010).

114. Nagata-Kuroiwa, R. et al. Critical Role of Neuropeptides B/W Receptor 1 Signaling in Social Behavior and Fear Memory. PLOS ONE 6, (2011). 
601

602

603

604

605

606

607

608

609

610

611

612

613

614

615

616

617

618

619

620

621

622

623

624

625

115. Tanaka, H. et al. Characterization of a family of endogenous neuropeptide ligands for the $G$ proteincoupled receptors GPR7 and GPR8. Proc. Natl. Acad. Sci. 100, 6251-6256 (2003).

116. Chen, Y. et al. Sustained NPY signaling enables AgRP neurons to drive feeding. eLife 8, (2019).

117. Watanabe, N. et al. A single nucleotide polymorphism of the neuropeptide B/W receptor-1 gene influences the evaluation of facial expressions. PloS One 7, e35390 (2012).

118. Kingsbury, M. A. \& Wilson, L. C. The Role of VIP in Social Behavior: Neural Hotspots for the Modulation of Affiliation, Aggression, and Parental Care. Integr. Comp. Biol. 56, 1238-1249 (2016).

119. Reiner, A. et al. Revised nomenclature for avian telencephalon and some related brainstem nuclei. J. Comp. Neurol. 473, 377-414 (2004).

120. Wilson, L. C., Goodson, J. L. \& Kingsbury, M. A. Seasonal Variation in Group Size Is Related to Seasonal Variation in Neuropeptide Receptor Density. Brain. Behav. Evol. 88, 111-126 (2016).

121. Schindelin, J. et al. Fiji: an open-source platform for biological-image analysis. Nat. Methods 9, 676-682 (2012).

122. McQuin, C. et al. CellProfiler 3.0: Next-generation image processing for biology. PLOS Biol. 16, e2005970 (2018).

123. Paxinos, G. \& Franklin, K. B. J. The mouse brain in stereotaxic coordinates. (Academic, 2004).

124. Paxinos, G. \& Franklin, K. B. J. Paxinos and Franklin's The mouse brain in stereotaxic coordinates. (Academic Press, an imprint of Elsevier, 2019).

125. Bogovic, J. A., Hanslovsky, P., Wong, A. \& Saalfeld, S. Robust Registration of Calcium Images by Learned Contrast Synthesis. 2016 IEEE 13th Int. Symp. Biomed. Imaging ISBI 1123-1126 (2016) doi:10.1109/ISBI.2016.7493463.

126. Conte, W. L., Kamishina, H. \& Reep, R. L. Multiple neuroanatomical tract-tracing using fluorescent Alexa Fluor conjugates of cholera toxin subunit B in rats. Nat. Protoc. 4, 1157-1166 (2009).

127. Hunter, J. D. Matplotlib: A 2D Graphics Environment. Comput. Sci. Eng. 9, 90-95 (2007).

128. Pedregosa, F. et al. Scikit-learn: Machine Learning in Python. J. Mach. Learn. Res. 12, 2825-2830 (2011). 
626 129. Gottman, J. M. \& Roy, A. K. Sequential Analysis: A Guide for Behavioral Researchers. (Cambridge

$627 \quad$ University Press, 1990).

628 130. Novák, P. \& Zahradník, I. Q-Method for High-Resolution, Whole-Cell Patch-Clamp Impedance

629 Measurements Using Square Wave Stimulation. Ann. Biomed. Eng. 34, 1201-1212 (2006).

630 131. Virtanen, P. et al. SciPy 1.0: fundamental algorithms for scientific computing in Python. Nat. Methods 17, $631261-272(2020)$.

632 132. Ward, J. H. Hierarchical Grouping to Optimize an Objective Function. J. Am. Stat. Assoc. 58, 236-244

633 (1963).

634 133. Michael Waskom et al. mwaskom/seaborn: v0.11.0 (Sepetmber 2020). (Zenodo, 2020).

635 doi:10.5281/zenodo.4019146.

636 
638 Fig. 1. DRN ${ }^{\text {DAT }}-$ CeA photostimulation promotes sociability but DRN ${ }^{\text {DAT }}$-BLP mediates place avoidance. a, $A A V_{5}$-DIO-ChR2-eYFP or $A_{A} V_{5}$-DIO-eYFP was injected into the DRN of DAT::Cre mice and optic fibers implanted over the BNST, CeA, or BLP to photostimulate DRNDAT terminals. After $>7$ weeks to allow for viral expression, cages of mice were assayed for social dominance using the tube test, prior to other behavioral tasks. $\mathbf{b}$, Heatmaps showing the relative location of ChR2-expressing mice in the three-chamber sociability assay, with optic fibers located over the BNST (left), CeA (center), or BLP (right). The task was repeated across two days, with one session paired with photostimulation ('ON') and one without ('OFF'). c, Photostimulation of DRNDAT-CeA terminals (8 pulses of 5 ms pulsewidth $473 \mathrm{~nm}$ light, delivered at $30 \mathrm{~Hz}$ every $5 \mathrm{~s}$ ) in ChR2-expressing mice (ChR2 ${ }^{\mathrm{CeA}}$ ) increased time spent in the social zone relative to the object zone ('social:object ratio'; paired t-test: $t_{28}=2.91$; corrected for multiple comparisons across projections: $p=0.021 ; n=29$ ), but had no significant effect on ChR2 ${ }^{\text {BNST }}$ mice (paired t-test: $t_{26}=0.552, p=0.586 ; n=27$ ), ChR2 ${ }^{B L P}$ mice (paired t-test: $t_{13}=1.62 . p=0.13, n=14$ ), nor eYFP mice. d, Scatter plots displaying relative dominance plotted against the change in social zone time with optical stimulation (ON-OFF), showing significant positive correlation in ChR2 ${ }^{\mathrm{CeA}}$ mice (Pearson's correlation: $r=0.549, p=0.002$ ). Inset bar graphs show mean values for subordinate, intermediate, and dominant mice. e, Example tracks of ChR2 ${ }^{\mathrm{BNST}}$, ChR2 ${ }^{\mathrm{CeA}}$, and $\mathrm{ChR} 2^{\mathrm{BLP}}$ mice in the real-time place preference (RTPP) assay, and bar graphs showing the difference in \% time spent in the stimulated ('ON') and unstimulated ('OFF') zones. ChR2 ${ }^{\mathrm{BLP}}$ mice spent proportionally less time in the stimulated zone relative to eYFP ${ }^{B L P}$ mice (unpaired t-test: $t_{13}=2.13, p=0.0455, n=14$ ChR2 ${ }^{B L P}, n=8$ eYFPBLP). f, Time spent in the ON zone across the 30 min session. ChR2 ${ }^{\mathrm{BLP}}$ mice spent significantly less time in the ON zone relative to eYFPBLP mice at $15 \mathrm{~min}$ (repeated measures two-way ANOVA: $F_{1,20}$ $=4.53$, main effect of opsin $p=0.046$, Bonferroni post-hoc tests ${ }^{*} p<0.05$ at $15 \mathrm{~min}$ ). $\mathbf{g}$, Scatter plots showing relative dominance plotted against the difference in zone time (insets show mean values for subordinate, intermediate, and dominant mice). Bar and line graphs display mean \pm SEM. ${ }^{*} p<0.05$, ${ }^{* *} p<0.01$.

Fig. 2. Collateralization of DRN ${ }^{\mathrm{DAT}}$ projections. a, The retrograde tracer cholera toxin subunit-B (CTB) conjugated to Alexa Fluor 555 (CTB-555, magenta) or Alexa-Fluor 647 (CTB-647, cyan) was injected into two downstream targets. $\mathbf{b}$, Confocal images showing representative injection sites for dual BNST and CeA injections (left panels), BNST and BLP (center panels), and CeA and BLP (right panels). c, High magnification images of DRN cells expressing CTB-555 (magenta), CTB-647 (cyan), and TH (green) following injection into the BNST and CeA. White arrows indicate triple-labelled cells. d, Venn diagrams showing the proportion of $\mathrm{CTB}+/ \mathrm{TH}+$ cells in the DRN following dual injections placed in the BNST and CeA (left), BNST and BLP (center), or CeA and BLP (right). When injections were placed in the BNST and CeA, dual CTB-labelled TH+ cells constituted $46 \%$ of all BNST projectors and $55 \%$ of all CeA projectors. In contrast, when injections were placed in the BNST and BLP, or CeA and BLP, the proportion of dual-labelled cells was considerably lower $(7.6 \%$ of BNST projectors and $9.7 \%$ of CeA projectors). e, Heatmaps indicating the relative density of $\mathrm{TH}+\mathrm{CTB}+$ cells throughout the DRN/CLi for each projector population and $\mathbf{f}$, dual-labelled cells. Color intensity represents average number of cells per slice. The total number of $\mathrm{TH}+$ BNST and CeA projectors per slice was similar $(n=27.9$ BNST projectors and $n=27.2 \mathrm{CeA}$ projectors per slice), whereas $\mathrm{TH}+\mathrm{BLP}$ projectors were significantly fewer in number ( $n=6.4$ BLP projectors per slice; Kruskal-Wallis statistic $=83.5, p<0.0001$; Dunn's post-hoc tests: BNST vs. CeA p>0.05, BNST vs BLP $p<0.001$, CeA vs BLP $p<0.001$ ). TH+ BNST and CeA projectors, and dual-labelled cells, were broadly distributed throughout the DRN, vIPAG, and CLi, with 
681

682

683

684

685

686

687

688

689

690

691

692

693

694

695

696

697

698

699

700

701

702

703

704

705

706

707

708

709

710

711

712

713

714

715

716

717

718

719

720

721

722

723

724

a higher concentration in the dorsal aspect of the DRN, whereas BLP projectors tended to be relatively denser in ventral DRN/CLi.

Fig. 3. Spatial-segregation of dopamine and neuropeptide receptor populations within DRNDAT terminal fields. a, Average image showing terminal density in the middle anteroposterior (AP) region of the BNST, following eYFP expression in DRNDAT (left) or VTA ${ }^{\text {DAT }}$ (right) neurons. b, Average images showing fluorescent puncta in the BNST indicating detection of Drd1 (red), Drd2 (yellow), Vipr2 (green), or Npbwr1 (blue) mRNA transcripts. c, Line graphs showing the percent of cells expressing each receptor ( $\geq 5$ puncta) across AP locations for the oval nucleus, dorsolateral BNST, and dorsomedial BNST (two-way ANOVA, oval nucleus: RNA probe $x$ AP interaction, $F_{9,160}=6.194, p<0.0001$, dorsolateral BNST: RNA probe $x$ AP interaction, $F_{12,167}=3.410, p=0.0002$, dorsomedial BNST: RNA probe $x$ AP interaction, $\left.\mathrm{F}_{12,161}=2.268, \mathrm{p}=0.0110\right)$. Drd1: $\mathrm{n}=51,55,53$, Drd2: $\mathrm{n}=52,55,53$, Vipr2: $\mathrm{n}=37,39,37$, Npbwr1: $\mathrm{n}=36,38,38$ sections, for oval nucleus, dorsolateral BNST, and dorsomedial BNST, respectively, from 4 mice. d, Matrices indicating overlap between mRNA-expressing cells: square color indicates the percent of cells expressing the gene in the column from within cells expressing the gene in the row. e, Average image showing terminal density in the middle AP region of the CeA, following eYFP expression in DRNDAT (left) or VTA ${ }^{\text {DAT }}$ (right) neurons. f, Average images showing fluorescent puncta in the CeA indicating mRNA expression. $\mathbf{g}$, Line graphs showing the $\%$ of cells expressing each receptor ( $\geq 5$ puncta) across AP locations for the CeL, CeM, and CeC (two-way ANOVA, CeL: RNA probe $x$ AP interaction, $F_{12,220}=8.664, p<0.0001$, CeM: main effect of RNA probe, $F_{3,186}=60.30, p<0.0001$, CeC: RNA probe $x$ AP interaction, $\left.F_{12,218}=4.883, p<0.0001\right)$. Drd1: $n=47,40,47$, Drd2: $\mathrm{n}=70,55,70$, Vipr2: $\mathrm{n}=65,57,63$, Npbwr1: $\mathrm{n}=62,50,60$ sections, for CeL, CeM, and CeC, respectively, from 4 mice. $\mathbf{h}$, Matrices indicating overlap between mRNA-expressing cells. i, Average image showing terminal density in the middle AP region of the BLP, following eYFP expression in DRNDAT (left) or VTA DAT (right) neurons. j, Average images showing fluorescent puncta in the BLP indicating $m R N A$ expression. $\mathbf{k}$, Line graphs showing the percent of cells expressing each receptor $(\geq 5$ puncta) across AP locations for the BLP and BMP (two-way ANOVA, BLP: RNA probe $x$ AP interaction, $F_{15,176}=2.165, p=0.0091$, BMP: main effect of RNA probe, $\left.F_{3,141}=56.92, p<0.0001\right)$. Drd1: $n=55,44$ Drd2: $\mathrm{n}=59,46$ Vipr2: $\mathrm{n}=41,33$ Npbwr1: $\mathrm{n}=45,34$ sections, for BLP and BMP, respectively, from 4 mice. I, Matrices indicating overlap between mRNA-expressing cells. Line graphs show mean $\pm \mathrm{SEM}$.

Fig. 4. DRN ${ }^{D A T}$ input influences downstream activity in a cell type-dependent manner. a, In mice expressing ChR2 in DRN ${ }^{D A T}$ neurons, ex vivo electrophysiological recordings were made from the BNST, b, CeA, and c, BLP. d, Photostimulation of DRNDAT terminals with blue light (8 pulses delivered at $30 \mathrm{~Hz}$ ) evoked both excitatory and inhibitory responses at resting membrane potentials in the BNST, $\mathbf{e}, \mathrm{CeA}$, and $\mathbf{f}, \mathrm{BLP}$. Traces show single sweeps and pie charts indicate proportion of cells with no response ('none'), an EPSP only ('excitation'), an IPSP only ('inhibition'), or a mixed response ('mix'). Recorded cells: BNST $n=19$, CeA $n=36, B L P n=48$, from 19 mice. $\mathbf{g}$, When constant current was injected to elicit spontaneous firing, BNST cells responded to photostimulation with an increase in firing ('excitation'), while $\mathbf{h}, \mathrm{CeA}$ and $\mathbf{i}, \mathrm{BLP}$ cells responded with an increase or a decrease in firing ('inhibition'). Recorded cells: BNST $n=5, \operatorname{CeA} n=20, B L P n=17$. j, Properties of the optically-evoked excitatory post-synaptic potential (EPSP) at resting membrane potentials - left: peak amplitude (Kruskal-Wallis statistic $=6.790, p=0.0335$; Dunn's posts-hoc tests: CeA vs BLP $p=0.0378$ ); middle: change in amplitude across light pulses; right: violin plots showing distribution of onset latencies (white circle indicates median). $\mathbf{k}$, Properties of the optically-evoked inhibitory post-synaptic potential (EPSP) 
725 at resting membrane potentials - left: trough amplitude (one-way ANOVA, $F_{2,31}=8.150, p=0.0014, C e A$ 726 vs BLP: $\left.{ }^{* *} \mathrm{p}=0.0014\right)$; middle: violin plot showing latency to trough peak; right: violin plot showing tau for 727 the current decay (white circle indicates median). Bar and line graphs display mean $\pm S E M$. ${ }^{*} p<0.05$, $728{ }^{* *} p<0.01$. I, Workflow for agglomerative hierarchical clustering of CeA neurons and $\mathbf{m}$, BLP neurons. 729 Four baseline electrical properties were used as input features, following max-min normalization (see 730 Methods) and Ward's method used to generate a cluster dendrogram, grouping cells based on

731 Euclidean distance. $\mathbf{n}$, Dendrogram for CeA cells indicating two major clusters, with their response to 732 DRN ${ }^{\text {DAT }}$ input indicated below each branch (excitation=black; inhibition=grey; no response=open). $\mathbf{0}$, 733 Upper panels: cluster 1 showed baseline properties typical of 'late-firing' neurons and cluster 2 showed 734 baseline properties typical of 'regular-firing' neurons. Lower panels: pie charts showing the response of 735 cells in each cluster to DRN ${ }^{D A T}$ input. $\mathbf{p}$, Dendrogram for BLP cells indicating two major clusters, with 736 their response to DRNDAT input indicated below each branch (excitation=black; inhibition=grey; no 737 response=open). q, Upper panels: cluster 1 showed baseline properties typical of pyramidal neurons 738 and cluster 2 showed baseline properties typical of GABA interneurons. Lower panels: pie charts 739 showing the response of cells in each cluster to DRN ${ }^{D A T}$ input. 
742 Fig. S1. DRN ${ }^{\mathrm{DAT}}$ and VTA ${ }^{\mathrm{DAT}}$ afferents target distinct downstream regions. Related to Fig. 1 and 743 Fig. 3. a-d, Confocal images at different AP locations through the VTA and DRN showing the typical spread of eYFP expression (green) following an injection of $A A V_{5}-D I O-C h R 2-e Y F P$ into a-b, the DRN and $\mathbf{c}-\mathbf{d}$, the VTA. Tyrosine hydroxylase ( $\mathrm{TH}$; the rate limiting enzyme in dopamine synthesis) expression from immunohistochemistry is shown in red. b,d, High magnification images of the substantia nigra pars compacta (SNc), VTA, rostral linear nucleus (RLi), caudal linear nucleus (CLi), and DRN. Viral injection in the DRN typically resulted in eYFP-expressing cells within the DRN, ventrolateral periaqueductal grey (vIPAG), and CLi nuclei, with minimal expression in the RLi, and none in the VTA or substantia nigra pars compacta (SNc). In contrast, viral injection in the VTA produced robust eYFP expression in SNc and VTA cell bodies, with some RLi expression, and none in the CLi, vIPAG, or DRN. e, Example images of downstream regions showing $\mathrm{TH}$ expression from immunohistochemistry and $\mathbf{f}$, eYFP expression in the prefrontal cortex (PFC), nucleus accumbens (NAc), bed nucleus of the stria terminalis (BNST), central amygdala (CeA), and posterior basolateral amygdala (BLP) following injection into the DRN (upper panels) and the VTA (lower panels). g, Quantification of mean eYFP fluorescence in subregions from each structure (PFC: $n=18$ and 14 sections, striatum: $n=20$ and 21 sections, BNST: $n=14$ and 13 sections, CeA: $n=24$ and 27 sections, amygdala: $n=45$ and 51 sections from DRN and VTA injections, respectively, from 6 mice). eYFP fluorescence was significantly greater following VTA injection in all striatal subregions (unpaired t-test: $\mathrm{CPu}: \mathrm{t}_{39}=13.23, \mathrm{p}<0.0001$; NAc core: $\mathrm{t}_{39}=13.56, p<0.0001$; NAc lateral shell: $\mathrm{t}_{31}=13.01, \mathrm{p}<0.0001 ; \mathrm{NAc}$ medial shell: $t_{37}=4.49, p<0.0001$ ), and significantly greater following DRN injection in the BNST oval nucleus (unpaired t-test: $t_{22}=3.95, p=0.0007$ ) and CeA lateral division (unpaired t-test: unpaired t-test: $\left.t_{34}=3.18, p=0.0031\right)$. $h$, Images from three selected downstream targets showing average terminal density in the middle anteroposterior (AP) region following eYFP expression in DRN ${ }^{D A T}$ (left) or VTA ${ }^{\text {DAT }}$ (right) neurons. Bar graphs show mean \pm SEM. PFC: $\mathrm{Cg}=$ cingulate cortex, $\mathrm{PL}=$ prelimbic cortex, IL= infralimbic cortex; striatum: $\mathrm{CPu}=$ caudate putamen, NAc core=nucleus accumbens core, NAc I.sh.=nucleus accumbens lateral shell, NAc m.sh.=nucleus accumbens medial shell; BNST: oval nuc.= BNST oval nucleus, lat.=BNST lateral division, med.=BNST medial division, vent. $=$ BNST ventral part; $\mathrm{CeL}=$ central amygdala lateral division, $\mathrm{CeM}$, central amygdala medial division, $\mathrm{CeC}=$ central amygdala capsular division; amygdala: $\mathrm{LA}=$ lateral amygdala, $\mathrm{BLA}=$ basolateral amygdala, $\mathrm{BLP}=$ basolateral amygdala posterior.

Fig. S2. Analysis of baseline behavioral traits and state transition in the juvenile intruder task. Related to Fig 1. a, Example confocal images showing ChR2-expressing DRN ${ }^{D A T}$ terminals and fiber placement over the BNST, b, CeA, and c, BLP. d, The tube test for social dominance was performed prior to optogenetic manipulations. e, Proportion of wins for an individual cage tested across four days, and average for all cages used in optogenetic manipulation experiments, separated by number of mice per cage (red=dominant, orange=intermediate, yellow=subordinate). $\mathbf{f}$, Correlation matrix indicating the relationship between baseline behavioral measures for all mice used in Fig. 1 and Fig. S3. For the open field test (OFT) and elevated-plus maze (EPM) the first $5 \mathrm{~min}$ of the task were used and for the juvenile intruder and 3 chamber assays the data from the 'OFF' session was used. g, Principal component analysis (PCA) of behavioral measures with point color representing the social dominance score for each animal. Inset, scree plot showing \% variance explained by the first 5 PCs. h-i, Home-cage behavior was assessed in the juvenile intruder assay across two counterbalanced sessions, one paired with photostimulation ('ON') and one without ('OFF'). DRN ${ }^{D A T}-C e A$ photostimulation in ChR2-expressing 
mice increased time spent engaged in face investigation with the juvenile mouse (paired t-test: $t_{22}=2.36$, $p=0.027$ ), whereas $\mathbf{j}-\mathbf{k}$, DRNDAT-BNST photostimulation increased time spent rearing (paired t-test: $\mathrm{t}_{23}=2,32, \mathrm{p}=0.0298$ ). I, A two-state Markov model was used to examine behavioral transitions during the juvenile intruder assay for DRNDAT-CeA mice. $m$, Bar graphs showing the difference in transition probability (ON-OFF) for within-state transitions and across-state transitions, for $\mathrm{ChR2}{ }^{\mathrm{CeA}}$ and $\mathrm{ChR} 2^{\mathrm{eYFP}}$ mice. There was no significant difference between ChR2 and eYFP groups for the change in withinstate transition probability (two-way ANOVA: opsin $x$ transition interaction, $F_{1,37}=3.149, p=0.0842$ ) or across-state transition probability (two-way ANOVA: opsin $x$ transition interaction, $F_{1,37}=0.542, p=0.466$ ) with photostimulation. Graphs show mean \pm SEM.

Fig. S3. Photostimulation of DRN ${ }^{\text {DAT }}$ projections does not modify locomotor or anxiety-like behavior, or support self-stimulation. Related to Fig 1. a, Example tracks in the open field test from a ChR2 ${ }^{\mathrm{BNST}}$, b, ChR2 ${ }^{\mathrm{CeA}}$, and $\mathbf{c}, \mathrm{ChR} 2^{\mathrm{BLP}}$ mouse. Photostimulation had no significant effect on time spent in the center of the open field (two-way ANOVA, light $x$ group interaction, BNST $-F_{2,94}=0.105$, $p=0.901 ; \mathrm{CeA}-\mathrm{F}_{2,98}=0.613, \mathrm{p}=0.544$; $\mathrm{BLP}-\mathrm{F}_{2,40}=0.181, \mathrm{p}=0.835$ ) or distance travelled (two-way ANOVA, light $x$ group interaction, BNST $-F_{2,94}=0.157, p=0.855$; CeA $-F_{2,98}=0.163, p=0.850$; BLP $F_{2,40}=0.252, p=0.771$ ) for DRNDAT-BNST, DRNDAT-CeA, or DRN ${ }^{D A T}$-BLP mice. $d$, Example tracks in the elevated plus maze (EPM) from a ChR2 ${ }^{\mathrm{BNST}}$, e, ChR2 ${ }^{\mathrm{CeA}}$, and $\mathbf{f}, \mathrm{ChR2} 2^{\mathrm{BLP}}$ mouse. Photostimulation had no significant effect on time spent in the open arms of the EPM (two-way ANOVA, light $x$ group interaction, $B N S T-F_{2,58}=0.896, p=0.414, C e A-F_{2,78}=0.160, p=0.852, B L P-F_{2,40}=0.354, p=0.704$ ) for DRNDAT_BNST, DRNDAT_CeA, or DRNDAT_BLP mice. $\mathbf{g}-\mathbf{i}$, Photostimulation of the DRNDAT_BNST, DRNDAT-CeA, or DRNDAT-BLP projection did not support intra-cranial self-stimulation (ICSS) as shown by a lack of preference for the active nosepoke (paired with blue light delivery) over the inactive nosepoke. $\mathbf{j}$, Scatter plots displaying the change in face investigation against the change in rearing with photostimulation (ON-OFF) for ChR2 ${ }^{\mathrm{BNST}}, \mathbf{k}, \mathrm{ChR} 2^{\mathrm{CeA}}$, l, and ChR2 ${ }^{\mathrm{BLP}}$ mice in the juvenile intruder assay. Outer plots are probability density curves, using kernel density estimation, to show the distribution of each behavior. Line and bar graphs show mean \pm SEM.

Fig. S4. Verification of dual-retrograde tracing strategy and intersectional approach to reveal axon collaterals. Related to Fig 2. a, Two retrograde tracers (CTB-555 and CTB-647) were injected into the same location, followed by sectioning and immunohistochemistry after 7 days. Right panels show example injection site for CTB-555 and CTB-647 in the BNST. b, CTB-expressing cells in the DRN with TH (green) revealed by immunohistochemistry. White arrows indicate triple-labelled cells. c, Within the $\mathrm{TH}+$ cells in the DRN, injection of both retrograde tracers into the same location resulted in 97\% CTB-647+ cells co-labelled with CTB-555, and 100\% CTB-555+ cells co-labelled with CTB-647. d, Injection strategy to enable eYFP expression selectively in the DRNDAT-CeA projection. A retrogradely-travelling HSV construct encoding mCherry-flpo, expressed in a Cre-dependent manner (HSV-LS1L-mCherry-IRES-flpo), was injected into the CeA of a DAT::Cre mouse, and an AAV, expressed in a flpo-dependent manner, encoding eYFP (AAV ${ }_{5}$ fDIO-eYFP) was injected into the DRN. e, After 7 weeks, this resulted in eYFP-expressing $\mathrm{TH}+$ cells in the DRN, and $\mathbf{f}$, eYFP-expressing processes in both the CeA (upper panels) and BNST (lower panels). g, Injection of only AAV $\mathrm{f}_{5} \mathrm{fDIO}$ eYFP into the DRN of a DAT::Cre mouse did not result in eYFP expression. 

pixels/cell and number of puncta/cell for three separate sections for each probe. c, Violin plots displaying puncta count per section for each receptor in the BNST (white circle indicates median; Drd1: $\mathrm{n}=51,55,53$ Drd2: $n=52,55,53$ Vipr2: $n=37,39,37$ Npbwr1: $n=36,38,38$ sections, for oval nucleus, dorsolateral BNST, and dorsomedial BNST, respectively, from 4 mice). d, Line graphs for each BNST subregion showing the number of expressing cells when using a threshold of 1 punctum/cell and $\mathbf{e}, 3$ puncta per cell. $\mathbf{f}$, Violin plots displaying puncta count per section for each receptor in the $\mathrm{CeA}$ (white circle indicates median; Drd1: $\mathrm{n}=47,40,47$ Drd2: $\mathrm{n}=70,55,70$ Vipr2: $\mathrm{n}=65,57,63$ Npbwr1: $\mathrm{n}=62,50,60$ sections, for CeL, $\mathrm{CeM}$, and $\mathrm{CeC}$, respectively, from 4 mice). $\mathbf{g}$, Line graphs for each CeA subregion showing the number of expressing cells when using a threshold of 1 punctum/cell and $\mathbf{h}, 3$ puncta per cell. $\mathbf{i}$, Violin plots displaying puncta count per section for each receptor in the amygdala (white circle indicates median; Drd1: $\mathrm{n}=55,44$ Drd2: $\mathrm{n}=59,46$ Vipr2: $\mathrm{n}=41,33$ Npbwr1: $\mathrm{n}=45,34$ sections, for BLP and BMP, respectively, from 4 mice). j, Line graphs for each amygdala subregion showing the number of expressing cells when using a threshold of 1 punctum/cell and $\mathbf{k}, 3$ puncta per cell. These lower thresholds yielded more expressing cells than using 5 puncta/cell (compare with Fig. 3c,g,k), but with a similar expression pattern across subregions and AP location. I-m, Example images showing expression of Vipr1 and Vipr2 within the BNST and CeA. We typically observed greater Vipr2 than Vipr1 expression, and high co-localization, and therefore concentrated our detailed analyses on Vipr2. Line graphs show mean \pm SEM.

Fig. S6. Effect of DRN ${ }^{\text {DAT }}$ photostimulation on downstream cellular excitability ex vivo. Related to Fig 4. a, Example DIC image, and corresponding eYFP fluorescence, of a brain slice containing the BNST, b, CeA, or c, BLP during ex vivo recording. Regional maps show the location of recorded cells, with color indicating the change in membrane potential elicited by optical stimulation of DRNDAT of the IPSP (lower panels) was maintained following application of TTX/4AP. e, Normalized peak amplitude of the EPSP and IPSP following TTX/4AP (EPSP, $n=8$; IPSP, $n=3$ ). f, Scatter plots showing the amplitude of the optically-evoked EPSP (left) and IPSP (right) recorded in downstream locations plotted against baseline membrane potential. g, Line graphs showing the action potential inter-event interval (IEI) in cells where constant current was injected to elicit firing. Raw (left) and normalized (right) IEI $5.5 \mathrm{~s}$ before and $5 \mathrm{~s}$ after optical stimulation of DRN ${ }^{\text {DAT }}$ terminals (blue shading) in BNST, CeA, and BLP cells. Cells which showed a reduction in IEI with optical stimulation were labelled 'excited' (excit., black) and cells which showed an increase in IEI with optical stimulation were defined as 'inhibited' (inhib., grey). h, Box-and-whisker plots comparing the baseline cell properties (used as input features for hierarchical clustering; Fig. 4l-q) of the two CeA clusters and $i$, the two BLP clusters. Unpaired $t-$ tests for $\mathrm{CeA}$ - ramp ratio: $\mathrm{t}_{24}=3.502, \mathrm{p}=0.0018$; max instantaneous firing frequency (max freq inst. $_{\text {. }}$ : $t_{24}=4.698, p<0.0001$, firing delay: $t_{24}=5.050, p<0.0001$, voltage sag: $t_{24}=3.983, p=0.0006$; unpaired $t-$ tests for BLP - capacitance: $t_{25}=4.803, p<0.0001$, max freq inst. $_{25}=15.48, p<0.0001$, firing delay: $t_{25}=2.743, p=0.0111$, voltage sag: $t_{25}=2.705, p=0.0121$. $j$, Box-and-whisker plots for the two CeA clusters and $\mathbf{k}$, the two BLP clusters showing the amplitude and latency of the EPSP and IPSP, and the combined total voltage area elicited by optical stimulation of DRNDAT terminals. EPSP peak amplitude, CeA: unpaired t-test, $t_{17}=1.40, p=0.180$; BLP: unpaired t-test $t_{22}=2.34, p=0.029$. EPSP latency, CeA: unpaired t-test, $t_{17}=0.673, p=0.510$; BLP: Mann-Whitney $U=33.5, p=0.032$. Total voltage area, CeA: 
871 Mann-Whitney $U=22, p=0.0023$; BLP: Mann-Whitney $U=29, p=0.0019$. I, Workflow for agglomerative 872 hierarchical clustering of all CeA and BLP neurons combined. Five cell properties were used as input 873 features, corresponding to the five used in Fig. 4I-q for separate clustering of CeA and BLP cells. $\mathbf{m}$, 874 Dendrogram indicating two major clusters, with the cell location and response to DRNDAT input indicated 875 by the colored bars below each branch (CeA - pink, BLP - blue; excitation=black; inhibition=grey; no 876 response=open). $\mathbf{n}$, Pie charts showing the response of cluster 1 and cluster 2 CeA cells (upper) and 877 BLP cells (lower) to optical stimulation DRNDAT input. ${ }^{*} p<0.05,{ }^{* *} p<0.01,{ }^{* * *} p<0.001,{ }^{* * *} p<0.0001$. 


\section{Animals and housing}

All procedures involving animals were conducted in accordance with $\mathrm{NIH}$ guidelines and approved by the MIT Committee on Animal Care or the Salk Institute Institutional Animal Care and Use

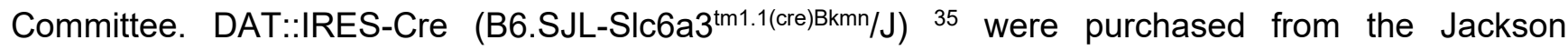
Laboratory (stock no. 006660; the Jackson Laboratory, ME, USA) and bred in-house to generate heterozygous male offspring for experiments. Wild-type C57BL/6J mice were purchased from Charles River Laboratories (MA, USA). Mice were housed on a $12 \mathrm{~h}: 12 \mathrm{~h}$ reverse light dark cycle (MIT: lights off 9am-9pm; Salk Institute: lights off 9.30am-9.30pm) with food and water available ad libitum. Mice were housed in groups of 2-4 with same-sex siblings.

\section{Surgery and viral constructs}

Mice (>7 weeks of age) were anaesthetized with isoflurane (inhalation: $4 \%$ for induction, $\sim 2 \%$ for maintenance, oxygen flow rate $1 \mathrm{~L} / \mathrm{min}$ ) before being placed in a digital small animal stereotax (David Kopf Instruments, CA, USA). Surgeries were performed under aseptic conditions with body temperature maintained by a heating pad throughout. Injections of recombinant adeno-associated viral (AAV) vectors, herpes simplex virus (HSV), or cholera toxin subunit-B (CTB) were performed using a beveled 33 gauge microinjection needle with a $10 \mu \mathrm{L}$ microsyringe (Nanofil; WPI, FL, USA). Virus or CTB was delivered at a rate of $0.1 \mu \mathrm{L} / \mathrm{min}$ using a microsyringe pump (UMP3; WPI, FL, USA) connected to a Micro4 controller (WPI, FL, USA). Following injection, the needle was maintained in place for $\sim 2 \mathrm{~min}$, then raised up by $0.05 \mathrm{~mm}$ and held for $\sim 10 \mathrm{~min}$ (to permit diffusion from the injection site) before being slowly withdrawn. Skull measurements were made relative to Bregma for all injections and implants. Implants were secured to the skull by a layer of adhesive cement (C\&B Metabond; Parkell Inc., NY, USA) followed by a layer of black cranioplastic cement (Ortho-Jet; Lang, IL, USA). Mice were given preemptive analgesia (1 mg/kg buprenorphine slow-release; sub-cutaneous; delivered concurrent with warmed Ringer's solution to prevent dehydration), supplemented with meloxicam (1.5 mg/kg; subcutaneous) where necessary, and were monitored on a heating pad until recovery from anesthesia.

$\mathrm{AAV}_{5}-\mathrm{EF} 1 \alpha-D I O-C h R 2-e Y F P, \quad A A V_{5}-E F 1 \alpha-D I O-e Y F P$, and $A A V_{5}-E F 1 a-f D I O-e Y F P$ were packaged by the University of North Carolina Vector Core (NC, USA) and received the AAV $\mathrm{F}_{5}$ EF1afDIO-eYFP construct from Karl Deisseroth and Charu Ramakrishnan. HSV-LS1L-mCherry-IRES-flpo was packaged by Dr. Rachael Neve at the Viral Gene Transfer Core Facility at MIT (now located at Massachusetts General Hospital).

\section{Immunohistochemistry and confocal microscopy}

Mice were deeply anaesthetized with sodium pentobarbital $(200 \mathrm{mg} / \mathrm{kg}$, intraperitoneal; IP) or euthasol $(150 \mathrm{mg} / \mathrm{kg}$; IP) followed by transcardial perfusion with $10 \mathrm{~mL}$ ice-cold Ringer's solution and $15 \mathrm{~mL}$ ice-cold 4\% paraformaldehyde (PFA). The brain was carefully dissected from the cranial cavity and immersed in 4\% PFA for $\sim 6-18 \mathrm{~h}$ before transfer to $30 \%$ sucrose solution in phosphate-buffered saline (PBS) at $4^{\circ} \mathrm{C}$. After at least $48 \mathrm{hr}$, brains were sectioned at $40 \mu \mathrm{m}$ on a freezing sliding microtome (HM430; Thermo Fisher Scientific, MA, USA) and sections stored at $4^{\circ} \mathrm{C}$ in $1 \mathrm{X}$ PBS. For immunohistochemistry, sections were blocked in PBS containing 0.3\% Triton X-100 (PBS-T; SigmaAldrich, MO, USA) with 3\% normal donkey serum (NDS; Jackson Immunoresearch, PA, USA) for 30$60 \mathrm{~min}$ at room temperature. This was followed by incubation in primary antibody solution (chicken anti$\mathrm{TH}$ (1:1000; AB9702; EMD Millipore, MA, USA) in 0.3\% PBS-T with $3 \%$ NDS) overnight at $4{ }^{\circ} \mathrm{C}$. Sections 
923

924

925

926

927

928

929

930

931

932

933

934

935

936

937

938

939

940

941

942

943

944

945

946

947

948

949

950

951

952

953

954

955

956

957

958

959

960

961

962

963

964

965

were then washed in 1X PBS four times (10 min each) before incubation in secondary antibody solution containing donkey anti-chicken 488 or 647 (1:1000; Jackson Immunoresearch, PA, USA) and a DNAspecific fluorescent probe (DAPI; 1:50000; Invitrogen, Thermo Fisher Scientific, MA, USA) in 0.2\% PBS$\mathrm{T}$ with $3 \%$ NDS for $1.5-2 \mathrm{hr}$ at room temperature. Sections were again washed four times in $1 \mathrm{X}$ PBS (10 min each) before being mounted on glass slides and coverslipped using warmed PVA-DABCO (Sigma-Aldrich, MO, USA).

Images were captured on a laser scanning confocal microscope (Olympus FV1000, Olympus, PA, USA) using Fluoview software version 4.0 (Olympus, PA, USA). Images were collected through a 10X/0.40 NA objective for injection site and optic fiber placement verification, a 20X/0.75 objective for terminal fluoresence quantification, and an oil-immersion 40X/1.30 NA objective for neurobiotin-filled neurons and RNAscope analysis (see individual Methods sections for more detail). FIJI ${ }^{121}$, CellProfiler 3.1 (Broad Institute, MA, USA) ${ }^{122}$, and Adobe Photoshop CC (Adobe Systems Incorporated, CA, USA) were used for subsequent image processing and analysis.

\section{Downstream fluorescence quantification}

In DAT::Cre mice, AAV 5 -EF1a-DIO-ChR2-eYFP (300 nL) was injected into the DRN (ML:1.20, AP:-4.10, DV:-2.90; needle at a $20^{\circ}$ angle from the midline, bevel facing medial) or VTA (ML:0.85, AP:2.70, DV:-4.50), and after 8 weeks mice underwent perfusion-fixation. Brains were subsequently sectioned at $40 \mu \mathrm{m}$, processed with immunohistochemistry for TH and DAPI, and serial z-stack images (3 $\mu \mathrm{m}$ optical thickness) collected at 20X on a confocal microscope. (See 'Immunohistochemistry and confocal microscopy' section above for details). A maximum projection was generated in FIJI and background subtraction based on the 'rolling ball' algorithm (radius $=50$ pixels) was applied to correct for uneven illumination. The appropriate brain atlas slice ${ }^{123,124}$ was overlaid onto the fluorescent image using the BigWarp plugin (https://imagej.net/BigWarp) ${ }^{125}$ in FIJI, by designating major anatomical landmarks based on DAPI staining and TH expression. Regions of interest (ROIs) were then annotated from the overlaid atlas, and mean fluorescence within each ROI quantified using FIJI. The PFC was examined from AP: 2.22 to 1.34, the striatum from AP 1.70 to 0.74 , the BNST from AP 0.37 to -0.11 , the CeA from AP -0.82 to -1.94 , and the amygdala from AP -0.82 to -2.92 . Average images in Fig. 3a, $3 \mathrm{e}, 3 \mathrm{i}$ and $\mathrm{S} 1 \mathrm{~h}$ were created by aligning individual images (from the middle AP region of the BNST, $\mathrm{CeA}$, or $\mathrm{BLP}$ ), using the line ROI registration plugin (https://imagej.net/Align Image by line ROI) in FIJI. An average projection was then performed across all images and the 'royal' LUT applied to visualize relative fluorescence intensity.

\section{Retrograde tracing and intersectional viral expression}

C57BL/6 mice were injected with 150-250 nL CTB conjugated to Alexa Fluor-555 (CTB-555) or Alexa Fluor-647 (CTB-647; Molecular Probes, OR, USA ${ }^{126}$ ) in two of three locations: the BNST (ML:1.10, AP:0.50, DV: -4.30; needle bevel facing back), CeA (ML:2.85, AP: -1.20; DV:-4.75; needle bevel facing back), or BLP (ML:3.35, AP:-2.20, DV:-5.25; needle bevel facing back). To assess retrograde CTB co-expression following injection of both fluorophore-conjugates of CTB into the same region (Fig. S4a-C), injections were either performed sequentially, or CTB-555 and CTB-647 were mixed prior to a single injection. After 7 days to allow for retrograde transport, mice were deeply anaesthetized with sodium pentobarbital $(200 \mathrm{mg} / \mathrm{kg}$ ) and perfused-fixed for subsequent histology. Brain sections containing injection sites and the DRN were prepared at $40 \mu \mathrm{m}$ and processed with immunohistochemistry for TH and DAPI. (See 'Immunohistochemistry and confocal microscopy' section above for details). CTB injection sites were verified with images acquired on a confocal microscope 
966

through a 10X objective (serial z-stack with $5 \mu \mathrm{m}$ optical thickness) and images of the DRN were acquired through a $40 \mathrm{X}$ objective (serial z-stack with $3 \mu \mathrm{m}$ optical thickness). DRN cells co-expressing CTB and TH were counted manually using the ROI 'point' tool in Fluoview software version 4.0 (Olympus, PA, USA). Counted files were imported into FIJI, and images overlaid onto the appropriate brain atlas image of the DRN using the BigWarp plugin (https://imagej.net/BigWarp) ${ }^{125}$. The $x-y$ coordinates of counted/marked $\mathrm{CTB}+/ \mathrm{TH}+$ cells were extracted using the 'Measure' function in FIJI. These coordinates were then used to generate heatmaps of cell location (Fig. 2e-f) by creating a 2D histogram using the Matplotlib package ${ }^{127}$ in Python .

Intersectional labelling of the dopaminergic projection from the DRN to the CeA was achieved by injecting HSV-LS1L-mCherry-IRES-flpo (300 nL) into the CeA (ML:2.85, AP:-1.45, DV:-4.55; needle bevel facing medial) and $\mathrm{AAV}_{5}$-fDIO-eYFP $(300 \mathrm{~nL})$ into the DRN (ML:1.20, AP:-4.10, DV:-2.90; needle at a $20^{\circ}$ angle from the midline, bevel facing medial) of a DAT::Cre mouse. After 8 weeks, mice were perfused-fixed with 4\% PFA, and the brain sectioned on a freezing microtome at $40 \mu \mathrm{m}$ before immunohistochemical processing with TH and DAPI. Images of eYFP-expressing cells in the DRN and terminals in the CeA and BNST were captured on a confocal microscope through a 20X objective with a serial z-section thickness of $3 \mu \mathrm{m}$.

\section{Behavioral assays and optogenetic manipulations}

DAT::Cre mice were injected with $300 \mathrm{~nL} A A V_{5}$ - EF1a-DIO-ChR2-eYFP or AAV 5 -EF1a-DIOeYFP in the DRN (ML:1.20; AP:-4.10; DV:-2.90; needle at a $20^{\circ}$ angle from the right side, bevel facing medial) and optic fibers (300 $\mu \mathrm{m}$ core, NA=0.37; Thorlabs, NJ, USA), held within a stainless steel ferrule (Precision Fiber Products, CA, USA), were implanted unilaterally or bilaterally over the BNST (unilateral: ML:1.10, AP:0.40, DV:-3.50; bilateral: ML:1.65, AP:0.40, DV:-3.35; $10^{\circ}$ angle from midline), CeA (ML:2.85, AP:-1.35, DV:-4.00), or BLP (ML:3.30, AP:-2.20, DV:-4.30). Behavioral experiments commenced 7-8 weeks following surgery. Mice were handled and habituated to patch cable connection once per day for at least 3 days before beginning optical manipulations. Behavioral testing was performed in dimly-lit soundproofed room during the mice's active dark phase ( 10am-5pm). On each testing day, mice were given at least $1 \mathrm{hr}$ to acclimate to the testing room before experiments began. For optical manipulations, optic fiber implants were connected to a patch cable via a ceramic sleeve (Precision Fiber Products, CA, USA), which itself was connected to a commutator (rotary joint; Doric, Québec, Canada) using an FC/PC adapter, to permit uninhibited movement. The commutator, in turn, was connected via a second patch cable (with FC/PC connectors) to a $473 \mathrm{~nm}$ diode-pumped solid state (DPSS) laser (OEM Laser Systems, UT, USA). To control the output of the laser, a Master-8 pulse stimulator (AMPI, Israel) was used, and the light power set to $10 \mathrm{~mW}$.

Tube test: Cages of mice (same-sex groups of 2-4) were assayed for social dominance using the tube test ${ }^{61,62}$. Mice were individually trained to pass through a clear Plexiglas tube $(30 \mathrm{~cm}$ length, $3.2 \mathrm{~cm}$ inner diameter) over 4 days. Each training trial involved releasing the mouse into the tube from one end, and ensuring it traveled through and out the other side. Mice which attempted to reverse or were reluctant to exit at the other end of the tube were gently encouraged forwards by light pressure from a plastic stick pressing on their hind region. Between trials mice freely explored the open arena outside tube $(76 \times 60 \mathrm{~cm}$ ) for $\sim 30-60 \mathrm{~s}$. Mice performed 8 training trials (4 from each end) on days 1 and 2 , and 3 trials (alternating ends) on days 3 and 4 . On days 5-8 mice competed against cagemates in a round-robin design. For each contest, mice were released simultaneously into opposite ends of the tube so that they met face-to-face in the center of the tube. The mouse which retreated from the confrontation was designated as the 'loser' and his opponent designated the 'winner'. Across testing 
1010

1011

1012

1013

1014

1015

1016

1017

1018

1019

1020

1021

1022

1023

1024

1025

1026

1027

1028

1029

1030

1031

1032

1033

1034

1035

1036

1037

1038

1039

1040

1041

1042

1043

1044

1045

1046

1047

1048

1049

1050

1051

1052

days, the side from which animals were released and the order in which they were tested against cagemates was counterbalanced. An animal's 'relative dominance' score reflected their proportion of 'wins' across all contests from 3-4 days of testing.

Open field test (OFT): The open field was composed of a square arena $(51 \times 51 \mathrm{~cm})$ made of transparent Plexiglas with $25 \mathrm{~cm}$ high walls. Mice freely explored the arena for $15 \mathrm{~min}$, and blue light (8 pulses with $5 \mathrm{~ms}$ pulse-width, at $30 \mathrm{~Hz}$, every $5 \mathrm{~s}$ ) was delivered during the middle 5 min epoch of the session. Animals were recorded using a video camera positioned above the arena, and Ethovision XT software used to track mouse location (Noldus, Netherlands). To assess anxiety-related behavior, for analysis, the chamber was divided into a 'center' square region and a 'periphery', with equal area.

Three-chamber sociability assay: The apparatus consisted of a $57.5 \mathrm{l} \times 22.5 \mathrm{w} \times 16.5 \mathrm{~h} \mathrm{~cm}$ chamber, with transparent Plexiglas walls and opaque grey plastic floors. The chamber was divided into unmarked left and right compartments (each $23 \times 22.5 \mathrm{~cm})$ and a smaller center compartment $(11.5 \times$ $22.5 \mathrm{~cm}$ ). An upturned wire mesh cup was placed in the left and right compartments. Each mouse first underwent a habituation session (10 $\mathrm{min}$ ) where they freely explored the chamber. They were then briefly $(\sim 1 \mathrm{~min})$ confined to the center compartment by the insertion of clear Plexiglas walls, while a novel object was placed under one of the two upturned cups, and a juvenile C57BL/6 mouse (3.5-5 weeks of age) was placed under the other upturned cup. The mice were then allowed to freely explore the chamber for a further $10 \mathrm{~min}$. The task was repeated on the second day, with the chamber rotated by $90^{\circ}$ relative to external spatial cues, and with a different novel object and novel juvenile mouse. The 10 min test epoch was paired with blue light delivery (8 pulses with 5 ms pulse-width, at $30 \mathrm{~Hz}$, every 5 s) on one of the two days, counterbalanced across animals. Mice were excluded if they showed a strong preference (>70\% time spent) for one side of the chamber in the habituation phase, or if they spent more than $1 \mathrm{~min}$ on top of the upturned cups during any session. Animals were recorded using a video camera positioned above the chamber and movement tracked using Ethovision XT (Noldus, Netherlands). The social:object ratio reflected the time spent in the 'social' side of the chamber (containing a novel juvenile mouse) divided by the time spent in the 'object' side of the chamber (containing a novel object).

Juvenile intruder assay: Mice were tested individually in their home cage. They freely explored alone for 5 min after which a novel juvenile mouse was placed in the cage for a further 3 min. The task was repeated on the second day with a different novel juvenile mouse. One of the two sessions was paired with blue light delivery (8 pulses with $5 \mathrm{~ms}$ pulse-width, at $30 \mathrm{~Hz}$, every $5 \mathrm{~s}$ ) which commenced after $2 \mathrm{~min}$ and continued until the end of the task ( $6 \mathrm{~min}$ total). The behavior of the mouse during the 3 min with the juvenile was scored manually using ODLog software (Macropod Software, Australia). Video files were scored twice (by two different observers, blinded to the experimental conditions) and the average of their counts was used for analysis. (See also 'First order Markov analysis' section).

Elevated plus maze (EPM): The EPM was made of grey plastic and consisted of two closed arms $(30 \mathrm{l} \times 5 \mathrm{w} \times 30 \mathrm{~h} \mathrm{~cm})$ and two open arms $(30 \mathrm{l} \times 5 \mathrm{w} \mathrm{cm})$, radiating at $90^{\circ}$ from a central platform (5 $x 5 \mathrm{~cm}$ ) and raised from the ground by $75 \mathrm{~cm}$. Mice freely explored for $15 \mathrm{~min}$, with blue light (8 pulses with $5 \mathrm{~ms}$ pulse-width, at $30 \mathrm{~Hz}$, every $5 \mathrm{~s}$ ) delivered during the middle 5 min epoch of the session. A video camera position above the EPM was used to record animals, and movement was tracked using Ethovision XT (Noldus, Netherlands).

Real-time place preference (RTPP): Mice were placed in a 52l x 52w x 26.5h cm transparent Plexiglas chamber, with clear panels separating left and right sides to leave a $11.5 \mathrm{~cm}$ gap for mice to 
1053

1054

1055

1056

1057

1058

1059

1060

1061

1062

1063

1064

1065

1066

1067

1068

1069

1070

1071

1072

1073

1074

1075

1076

1077

1078

1079

1080

1081

1082

1083

1084

1085

1086

1087

1088

1089

1090

1091

1092

1093

1094

1095

pass through. Mice freely explored for $30 \mathrm{~min}$, during which entry into one side of the chamber resulted in delivery of blue light ( 15 pulses with $5 \mathrm{~ms}$ pulse-width, at $30 \mathrm{~Hz}$, every $5 \mathrm{~s}$ ), which continued until mice exited the zone. Entry into the opposite side did not result in blue light delivery. The side paired with blue light delivery was counterbalanced across animals. A video camera positioned above the arena recorded animals, and mouse movement was tracked using Ethovision XT (Noldus, Netherlands).

Intra-cranial self-stimulation (ICSS): Mice were food deprived for 16-20 hr prior to each day of ICSS, in order to encourage behavioral responding. Testing was conducted in an operant chamber (Med Associates, VT, USA) within a custom sound-attenuating outer box. The operant chamber contained two illuminated nose-poke ports, each with an infrared beam, and a cue light positioned above each port. White noise was delivered continuously throughout the session, and successful nose-pokes (signaled by a beam break) resulted in an auditory tone (1 s duration, 1 or $1.5 \mathrm{kHz}$ ) and illumination of the respective cue light. A nose-poke at the 'active' port also triggered delivery of blue light ( 90 pulses with $5 \mathrm{~ms}$ pulse-width, at $30 \mathrm{~Hz}$ ) while a nose-poke at the 'inactive' port did not trigger light delivery. The physical location of the active and inactive nose-poke ports, and the auditory tone frequency associated with each port, was counterbalanced across animals. On day 1 (training), mice completed a $2 \mathrm{hr}$ session in the operant chamber in which both nose-poke ports were baited with a small amount of palatable food, in order to encourage investigation. On day 2 (testing), mice completed an identical $2 \mathrm{hr}$ session, except the nose-poke ports were not baited. Nose-poke activity was recorded with MedPC software (Med Associates, VT, USA) and analyzed using MATLAB (Mathworks, MA, USA). Only data from day 2 was used for analysis.

Analysis of baseline behavior: The baseline behavior of all mice (i.e. without stimulation) was evaluated to uncover any relationships between specific types of behavior assessed in different tasks. These analyses used relative dominance from the tube test, the first $5 \mathrm{~min}$ of the OFT and EPM, and the OFF trial from the three-chamber sociability and juvenile intruder assays. Correlation matrices were generated in GraphPad Prism 8 (GraphPad Software, CA, USA) to show the Pearson's correlation coefficient for each pair of variables.

Dimensionality reduction was performed on baseline behavior data using principal component analysis (PCA) with the scikit-learn module ${ }^{128}$ in Python. The eight input measures from behavioral assays were (1) percent time moving in the OFT, (2) time in the center of the OFT, (3) time in the open arms of the EPM, (4) social:object ratio in the three-chamber assay, and (5) time spent face sniffing, (6) anogenital sniffing, (7) rearing, and (8) grooming in the juvenile intruder assay. The data was first normalized to generate a covariance matrix and then the first $5 \mathrm{PCs}$ were extracted. Relative dominance was concatenated with the resulting PC values for each mouse to color-code individual points in the PC1 vs PC2 plot.

First order Markov analysis: Behavioral videos from the juvenile intruder assay were manually annotated so that each second of the $180 \mathrm{~s}$ session was assigned a code(s) from 15 behavioral categories:

- Social behaviors: face sniff (reciprocated), face sniff (non-reciprocated), flank sniff, anogenital sniff (reciprocated), anogenital sniff (non-reciprocated), close follow, approach, dominant climb, attack.

- Nonsocial behaviors: groom, dig, rear, climb, still, ambulate.

If more than one behavior occurred during a $1 \mathrm{~s}$ period, multiple behaviors were assigned to that second (up to a maximum of 3 ) to ensure all behavioral transitions were included. We considered two 
1096

1097

1098

1099

1100

1101

1102

1103

1104

1105

1106

1107

1108

1109

1110

1111

1112

1113

1114

1115

1116

1117

1118

1119

1120

1121

1122

1123

1124

1125

1126

1127

1128

1129

1130

1131

1132

Markov models - one 15-state model and one 2-state model, in which behaviors were assigned to either the 'social' or 'nonsocial' categories. For each animal, we created a transition probability matrix from each sequence by counting the number of transitions that occurred and dividing by the total number of occurrences of that behavior. To compute the overall transition probability matrix for the eYFP and ChR2 groups, we took the mean of the transition probability across all individuals in that group. Difference scores between the stimulation OFF and ON sessions were calculated by taking the difference across pairs of transition probability matrices corresponding to each individual, then calculating the mean across eYFP or ChR2-expressing mice.

To verify that a first order Markov model was an appropriate fit for our data we computed the log likelihood chi squared statistic ${ }^{129}$ :

$$
G=2 \sum_{j} \sum_{i} O_{i j} \ln \frac{O_{i j}}{E_{i j}},
$$

where $O_{i j} \geq 0$ is the observed number of transitions from state $\mathrm{i}$ to $\mathrm{j}, E_{i j} \geq 0$ is the expected number of transitions from state $i$ to state $j$ assuming a zeroth order Markov (i.e., no time dependence). We found that $\mathrm{G}$ was statistically significant for all subjects in both the 15 state and 2 state models, thus rejecting the null hypothesis of randomly transitioning between states.

We also tested whether a non-stationary model was a better fit for the data than a stationary model. To do this, we divided each subject's behavioral sequence into two segments of equal duration and computed transition probability matrices for each segment. We then computed a variation on the likelihood ratio chi square statistic ${ }^{129}$ :

$$
L R X=2 \sum_{s} \sum_{j} \sum_{i} f_{i j s} \ln \frac{\bar{p}_{i j s}}{p_{i j}},
$$

where $s$ represents the segment, $p_{i j}$ is the probability of transition from state $i$ to $j$ taken over the entire sequence, $\bar{p}_{i j s}$ is the probability of transition from $i$ to $j$ for each segment, and $f_{i j s}$ is the number of transitions from state $i$ to $j$ for each segment. Since not all subjects had a significant difference, we determined that a stationary model was the most appropriate model to fit all our data.

\section{Ex vivo electrophysiology}

DAT::Cre mice received an injection of $300 \mathrm{~nL}$ AAV 5 -DIO-ChR2-eYFP in the DRN (ML:1.20, AP:-4.10, DV:-2.90; needle at a $20^{\circ}$ angle from the midline, bevel facing medial), and after at least 8 weeks for transgene expression, mice were deeply anaesthetized with sodium pentobarbital (200 $\mathrm{mg} / \mathrm{kg}$ ) or euthasol $(150 \mathrm{mg} / \mathrm{kg} ; \mathrm{IP})$. They were then transcardially perfused with ice-cold $\left(\sim 4^{\circ} \mathrm{C}\right)$ modified artificial cerebrospinal fluid (ACSF; composition in $\mathrm{mM}: \mathrm{NaCl} 87, \mathrm{KCl} 2.5, \mathrm{NaH} 2 \mathrm{PO} 4^{*} \mathrm{H} 20$ 1.3, $\mathrm{MgCl}^{*} 6 \mathrm{H} 2 \mathrm{O} 7, \mathrm{NaHCO} 325$, sucrose 75 , ascorbate 5, $\mathrm{CaCl}{ }^{*} 2 \mathrm{H} 2 \mathrm{O} 0.5$, in ddH20; osmolarity $320-330$ mOsm, pH 7.30-7.40), saturated with carbogen gas (95\% oxygen, $5 \%$ carbon dioxide) before the brain was rapidly and carefully extracted from the cranial cavity. Thick coronal $(300 \mu \mathrm{m})$ slices containing the BNST, CeA, BLP, and DRN were prepared on a vibrating blade vibratome (VT1200; Leica Biosystems, Germany), in ice-cold modified ACSF saturated with carbogen gas. Brain slices were hemisected with a scalpel blade before transfer to a holding chamber containing ACSF (composition in $\mathrm{mM}$ : $\mathrm{NaCl} 126$, 

298-302 mOsm, $\mathrm{pH} 7.30-7.40)$ saturated with carbogen, in a warm water bath $\left(\sim 30^{\circ} \mathrm{C}\right)$.

Electrophysiological recordings were commenced after the slices had rested for at least $45 \mathrm{~min}$. During recording, the brain slice was maintained in a bath with continuously perfused ACSF, saturated with carbogen, at $31 \pm 1^{\circ} \mathrm{C}$ using a peristaltic pump (Minipuls3; Gilson, WI, USA). Slices were visualized through an upright microscope (Scientifica, UK) equipped with infrared-differential interference contrast (IR-DIC) optics and a Q-imaging Retiga Exi camera (Q Imaging, Canada). In the BNST, CeA, and BLP, recordings were performed in the region containing fluorescent DRNDAT terminals (expressing ChR2eYFP) with neurons visualized through a 40X/0.80 NA water immersion objective. Terminal expression was confirmed by brief illumination from a $470 \mathrm{~nm}$ LED light source (pE-100; CoolLED, NY, USA) combined with the appropriate filter set. Borosilicate glass capillaries were shaped on a P-97 puller (Sutter Instrument, CA, USA) to produce pipettes for recording that had resistance values of 3.5-5 MOhm when filled with internal solution (composition in $\mathrm{mM}$ : potassium gluconate $125, \mathrm{NaCl} 10, \mathrm{HEPES}$ 20, MgATP 3, and 0.1\% neurobiotin, in ddH20 (osmolarity $287 \mathrm{mOsm}$; pH 7.3). Whole-cell patch-clamp recordings were made using pClamp 10.4 software (Molecular Devices, CA, USA), with analog signals amplified using a Multiclamp $700 \mathrm{~B}$ amplifier, filtered at $3 \mathrm{kHz}$, and digitized at $10 \mathrm{kHz}$ using a Digidata 1550 (Molecular Devices, CA, USA). A $5 \mathrm{mV}, 250 \mathrm{~ms}$ hyperpolarizing step was used to monitor cell health throughout the experiment, and recordings were terminated if significant changes $(>20 \%)$ occurred to series resistance $\left(R_{s}\right)$, input resistance $\left(R_{\text {in }}\right)$, or holding current.

Passive cell properties (capacitance, membrane resistance) were estimated from the current response to hyperpolarizing $5 \mathrm{mV}, 250 \mathrm{~ms}$ steps, delivered in voltage-clamp from a holding potential of $-70 \mathrm{mV}$, using custom MATLAB code written by Praneeth Namburi, based on MATLAB implementation of the Q-Method ${ }^{130}$. To examine the membrane potential response to current injection, cells were recorded in current-clamp mode, and a series of $1 \mathrm{~s}$ steps were delivered, in $20 \mathrm{pA}$ increments, from $120 \mathrm{pA}$ to $260 \mathrm{pA}$. The voltage sag amplitude (attributable to the hyperpolarization-activated cation current; $I_{h}$ ) was measured as the difference between the peak instantaneous and steady-state membrane potential elicited during a $-120 \mathrm{pA}$ step (see Fig. 4I). The ramp ratio was calculated by dividing the average membrane potential between $900-1000 \mathrm{~ms}$ by the membrane potential between 100-200 ms following step onset, using the largest current step that elicited a subthreshold response (i.e. did not evoke action potentials). The firing delay was taken as the time between current step onset and the first elicited action potential, on delivery of the first current step that was elicited a suprathreshold response (i.e. rheobase current). The max instantaneous firing frequency (max freq.inst) was taken as the maximum firing frequency attained during the first $100 \mathrm{~ms}$ of the depolarizing current steps.

To photostimulate ChR2-expressing DRN ${ }^{\mathrm{DAT}}$ terminals in the BNST, CeA, and BLP, $470 \mathrm{~nm}$ light was delivered through the 40X/0.8 NA objective from an LED light source ( $p E-100$; CoolLED, NY, USA). Neurons were recorded at their resting membrane potential in current-clamp mode, and $470 \mathrm{~nm}$ light (8 pulses at $30 \mathrm{~Hz}, 5 \mathrm{~ms}$ pulse-width) was delivered every $30 \mathrm{~s}$. In a minority of cells which showed spontaneous activity at the resting potential, negative current was injected to hold the cell at a subthreshold potential (typically $\sim-60 \mathrm{mV}$ ). The peak amplitude of the optically-evoked excitatory postsynaptic potential (EPSP) or trough amplitude of the inhibitory post-synaptic potential (IPSP) was measured from the average trace using Clampfit 10.7 (Molecular Devices, CA, USA), using the $5 \mathrm{~s}$ prior to stimulation as baseline. Tau for the decay phase of the IPSP was estimated by fitting the IPSP with a single exponential, from the IPSP trough until return to baseline. Total voltage area was calculated 
1177 from 0-5.5 s following the onset of the first light pulse. In cells where optical stimulation evoked only an 1178 EPSP the response was classed as an 'excitation', only an IPSP was classed as an 'inhibition', and a 1179 combined optically-evoked EPSP and IPSP was classed as 'mixed'. To assess the effect of 1180 photostimulation on firing activity, constant positive current was injected to elicit consistent spontaneous 1181 action potentials, and $470 \mathrm{~nm}$ light (8 pulses at $30 \mathrm{~Hz}, 5 \mathrm{~ms}$ pulse-width) was delivered every $30 \mathrm{~s}$. The interevent interval (IEI) between action potentials was calculated for $5 \mathrm{~s}$ before and $5 \mathrm{~s}$ after the first pulse of blue light using Clampfit 10.7 (Molecular Devices, CA, USA). A decrease in IEI (indicating an increase in firing rate) was classed as an 'excitation' and an increase in IEI (indicating a decrease in firing rate) was classed as an 'inhibition'.

Following recording, images showing the location of the recording pipette within the slice were captured through a $4 \mathrm{X} / 0.10 \mathrm{NA}$ objective. Images were subsequently overlaid onto the appropriate brain atlas image ${ }^{123,124}$, recorded cell locations were annotated, and then converted into $x-y$ coordinates in FIJI. Python was used to generate a scatter plot of cell location, with points color-coded by the overall membrane potential response to photostimulation (Fig. S6a-c).

Unsupervised agglomerative hierarchical clustering was used to classify cells according to their baseline electrophysiological properties. This approach organizes objects (in this case cells) into clusters, based on their similarity. The electrophysiological properties used as input features for clustering $\mathrm{CeA}$ cells were ramp ratio, max firing frequency, firing delay, and voltage sag, which are characteristics that have been previously shown to distinguish between subtypes of CeA neuron ${ }^{103}$. For clustering BLP cells, we replaced ramp ratio with capacitance, as this measure is often used to distinguish between pyramidal neurons and GABAergic interneurons, which are the two main cell types in this region. Data for each cell property was max-min normalized to produce a $4 \times n$ matrix of input features (where $n=$ total number of cells). Clustering was performed using the 'linkage' function of SciPy 131 in Python, using Ward's linkage method ${ }^{132}$ and Euclidean distance. Briefly, this approach begins with each cell assigned to a single cluster. Cells that are in closest proximity (i.e. have highest similarity) are then linked to form a new cluster. Then the next closest clusters are linked, and so on. This process is repeated until all cells are included in a single cluster. The output of this analysis is plotted as a hierarchical tree (dendrogram), in which each cell is a 'leaf' and the Euclidean distance on the y-axis indicates the linkage between cells (larger distance indicates greater dissimilarity). To annotate the photostimulation response of cells on the dendrogram (Fig. $4 \mathrm{n}, 4 \mathrm{p}$, and $\mathrm{S6m}$ ), the response was designated as 'excitation' if action potential IEI decreased with optical stimulation and 'inhibition' if action potential IEI increased on stimulation. If firing data was not available, cells were designated as showing an 'excitation' if only an EPSP was evoked on optical stimulation, and 'inhibition' if only an IPSP was evoked. In cells where a mixed EPSP/IPSP was elicited, the response was designated as an 'excitation' if the overall voltage area (0-5.5 s following light onset) was positive, and an 'inhibition' if the overall voltage area was negative.

At the end of recording, brain slices were fixed in 4\% PFA overnight, and then washed in $1 \mathrm{X}$ PBS (4 $\times 10$ min each). Slices were blocked in 0.3\% PBS-T (Sigma-Aldrich, MO, USA) with $3 \%$ NDS (Jackson Immunoresearch, PA, USA) for 30-60 min at room temperature. They were then incubated in PBS-T 0.3\% with, 3\% NDS, and CF405- or CF633-conjugated streptavidin (1:1000; Biotium, CA, USA) for $90 \mathrm{~min}$ at room temperature to reveal neurobiotin labelling. Slices were finally washed four times in 1X PBS (10 min each) before being mounted on glass slides and coverslipped using warmed PVADABCO (Sigma-Aldrich, MO, USA).

\section{Single molecule fluorescent in situ hybridization (smFISH) with RNAscope}


C57BL/6 mice were deeply anesthetized with $5 \%$ isoflurane and brains were rapidly extracted and covered with powdered dry ice for $\sim 2 \mathrm{~min}$. Frozen brains were stored in glass vials at $-80^{\circ} \mathrm{C}$ before sectioning at $20 \mu \mathrm{m}$ using a cryostat (CM3050 S; Leica Biosystems, Germany) at $-16^{\circ} \mathrm{C}$. Coronal sections were thaw-mounted onto a glass slide, by gentle heating from the underside using the tip of a finger to encourage adhesion of the section to the slide. They were then stored at $-80^{\circ} \mathrm{C}$ until processing.

Fluorescent in situ hybridization (FISH) was performed using the RNAscope Multiplex Fluorescent assay v2 (Advanced Cell Diagnostics, CA, USA). The following products were used: RNAscope Multiplex Fluorescent Reagent Kit V2 (Catalog \#323110), Fluorescent Multiplex Detection Reagents (\#323110), target probes for Mus musculus genes - Drd1a (\#406491-C1), Drd2 (\#406501C3), Npbwr1 (\#547181-C1), and Vipr2 (465391-C2) - and the Tyramide Signal Amplification (TSA) Plus Fluorescence Palette Kit (NEL760001KT; PerkinEImer Inc., MA, USA) with fluorophores diluted to 1:1000-1:5000. The protocol was performed as recommended by the manufacturer, with some modifications to prevent tissue degradation and optimize labelling specificity in our regions of interest. Fresh frozen slices were fixed in $4 \%$ PFA for $1 \mathrm{hr}$ at $4^{\circ} \mathrm{C}$. Slices were dehydrated in an ethanol series $(50 \%, 70 \%, 100 \%$, and $100 \%$ ethanol, $5 \mathrm{~min}$ each) and then incubated in hydrogen peroxide for $8 \mathrm{~min}$ at room temperature. Protease treatment was omitted in order to prevent tissue degradation. Slides were then incubated with the desired probes (pre-warmed to $40^{\circ} \mathrm{C}$ and cooled to room temperature) for $2 \mathrm{hr}$ at $40^{\circ} \mathrm{C}$ in a humidified oven. Following washing $(2 \times 30 \mathrm{~s}$ in 1 X RNAscope wash buffer), signal amplification molecules (Amp 1, 2, and 3) were hybridized to the target probes in sequential steps, with 30 min incubation for Amp 1 and 2 and 15 min incubation for Amp 3 at $40^{\circ} \mathrm{C}$, all in a $40^{\circ} \mathrm{C}$ humidified oven followed by washing ( $2 \times 30 \mathrm{~s}$ in wash buffer). For fluorescent labelling of each amplified probe, slides were incubated in channel-specific HRP for $10 \mathrm{~min}$, followed by incubation with TSA fluorophore (PerkinElmer, MA, USA) for $20 \mathrm{~min}$, and then incubation in HRP-blocker for 10 minutes (with 2 x $30 \mathrm{~s}$ washes between each step). Probes for Drd1a, Drd2, Npbwr1, and Vipr2 were each labelled with green (TSA Plus Fluorescein), red (TSA Plus Cyanine 3), or far red (TSA Plus Cyanine 5) fluorophores in counterbalanced combinations. Slides were then incubated in DAPI (Advanced Cell Diagnostics, CA, USA) for $10 \mathrm{~min}$, washed in 1X RNAscope wash buffer, dried for $20 \mathrm{~min}$, coverslipped with warmed PVA-DABCO, (Sigma-Aldrich, St. Louis, MO) and left to dry overnight before imaging.

Images were captured on a confocal laser scanning microscope (Olympus FV1000; Olympus, PA, USA) using a 40X/1.30NA oil immersion objective. Serial Z-stack images were acquired using FluoView software version 4.0 (Olympus, PA, USA) at an optical thickness of $1.5 \mu \mathrm{m}$. All images were acquired with identical settings for laser power, detector gain, and amplifier offset. A maximum Zprojection was performed in FIJI followed by rolling ball background subtraction to correct for uneven illumination. Image brightness and contrast were moderately adjusted using FIJI, with consistent adjustments made across images for each probe-fluorophore combination. Regions of interest were annotated on each image by overlaying the appropriate brain atlas image ${ }^{123,124}$ with guidance from DAPI staining and using the BigWarp plugin (https://imagej.net/BigWarp) ${ }^{125}$ in FIJI. These ROI outlines were used to generate binary masks in order to regionally-restrict subsequent image analysis. Automated cell identification and analysis of fluorescent mRNA labelling was performed in CellProfiler 122 using a modified version of the 'Colocalization' template pipeline (https://cellprofiler.org/examples). The pipeline was optimized to identify DAPI labelling (20-40 pixels in diameter), in order to define cell outlines. This was followed by identification of fluorescent mRNA puncta (2-10 pixels in diameter) for each probe. Puncta that were localized within DAPI-identified cells (classified using the 'relate objects' module) were assigned to that cell for subsequent analysis. Quantification and further analysis/data 
1265 visualization was performed using a custom-written Python code. Violin plots were made using the violin 1266 plot function in the Seaborn library ${ }^{133}$ of Python (with smoothing set to 0.2 ), and colocalization matrices 1267 were generated using the Seaborn heatmap function.

\section{Statistical analyses}

$1269 \quad$ Statistical tests were performed using GraphPad Prism 8 (GraphPad Software, CA, USA). 1270 Normality was evaluated using the D'Agostino-Pearson test, and data are expressed as mean \pm standard 1271 error of the mean (SEM), unless otherwise noted. Data which followed a Gaussian distribution were 1272 compared using a paired or unpaired t-test (non-directional) for two experimental groups, and a one1273 way or two-way ANOVA with repeated measures for three or more experimental groups. Data for two 1274 experimental groups which did not follow a Gaussian distribution were compared using a Mann-Whitney $1275 U$ test. Correlation between two variables was assessed using the Pearson's product-moment 1276 correlation coefficient. Threshold for significance was set at ${ }^{*} p<0.05,{ }^{* *} p<0.01$ and ${ }^{* * *} p<0.001$. 


\section{Figures}

Figure 1
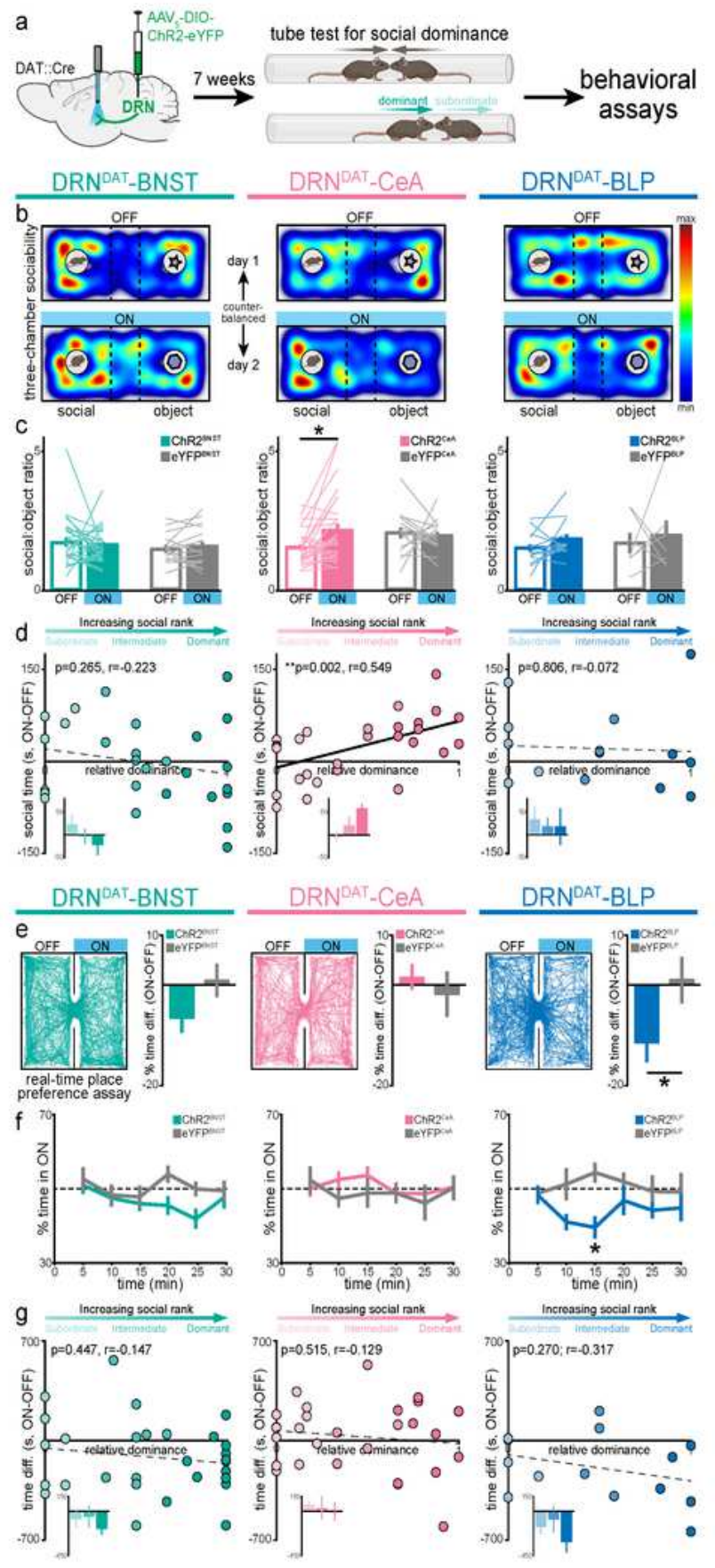

Figure 1

DRNDAT-CeA photostimulation promotes sociability but DRNDAT-BLP mediates place avoidance. a, AAV5DIO-ChR2-eYFP or AAV5-DIO-eYFP was injected into the DRN of DAT::Cre mice and optic fibers implanted over the BNST, CeA, or BLP to photostimulate DRNDAT terminals. After $>7$ weeks to allow for viral 
expression, cages of mice were assayed for social dominance using the tube test, prior to other behavioral tasks. $b$, Heatmaps showing the relative location of ChR2-expressing mice in the threechamber sociability assay, with optic fibers located over the BNST (left), CeA (center), or BLP (right). The task was repeated across two days, with one session paired with photostimulation ('ON') and one without ('OFF'). c, Photostimulation of DRNDAT-CeA terminals (8 pulses of $5 \mathrm{~ms}$ pulse-width $473 \mathrm{~nm}$ light, delivered at $30 \mathrm{~Hz}$ every $5 \mathrm{~s}$ ) in ChR2-expressing mice (ChR2CeA) increased time spent in the social zone relative to the object zone ('social:object ratio'; paired t-test: t28 $=2.91$; corrected for multiple comparisons across projections: $p=0.021 ; n=29$ ), but had no significant effect on ChR2BNST mice (paired t-test: $\mathrm{t} 26=0.552, p=0.586 ; n=27$ ), ChR2BLP mice (paired t-test: $\mathrm{t} 13=1.62 . p=0.13, \mathrm{n}=14$ ), nor eYFP mice. $d$, Scatter plots displaying relative dominance plotted against the change in social zone time with optical stimulation (ON-OFF), showing significant positive correlation in ChR2CeA mice (Pearson's correlation: $r=0.549, p=0.002$ ). Inset bar graphs show mean values for subordinate, intermediate, and dominant mice. e, Example tracks of ChR2BNST, ChR2CeA, and ChR2BLP mice in the real-time place preference (RTPP) assay, and bar graphs showing the difference in \% time spent in the stimulated ('ON') and unstimulated ('OFF') zones. ChR2BLP mice spent proportionally less time in the stimulated zone relative to eYFPBLP mice (unpaired t-test: $\mathrm{t} 13=2.13, \mathrm{p}=0.0455, \mathrm{n}=14$ ChR2BLP, $\mathrm{n}=8$ eYFPBLP). $\mathrm{f}$, Time spent in the ON zone across the 30 min session. ChR2BLP mice spent significantly less time in the $\mathrm{ON}$ zone relative to eYFPBLP mice at $15 \mathrm{~min}$ (repeated measures two-way ANOVA: F1,20 $=4.53$, main effect of opsin $p=0.046$, Bonferroni post-hoc tests ${ }^{*} p<0.05$ at $15 \mathrm{~min}$ ). $\mathrm{g}$, Scatter plots showing relative dominance plotted against the difference in zone time (insets show mean values for subordinate, intermediate, and dominant mice). Bar and line graphs display mean \pm SEM. ${ }^{\star} p<0.05,{ }^{* \star} p<0.01$. 
Figure 2

a
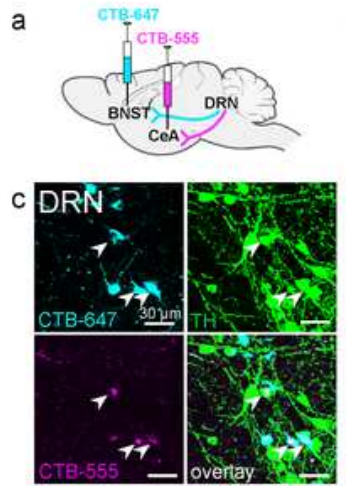

e
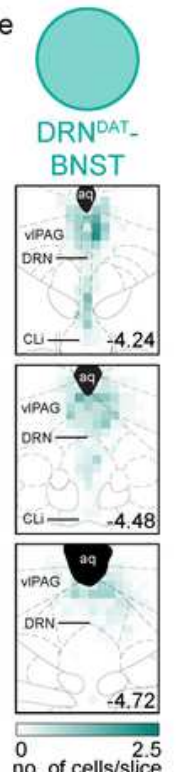

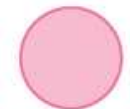

DRN ${ }^{\text {DAT_ }}$
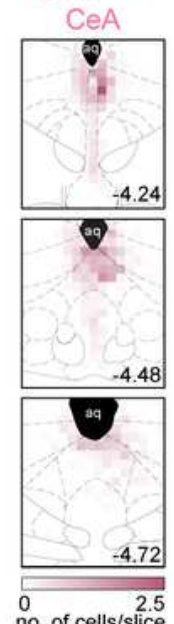
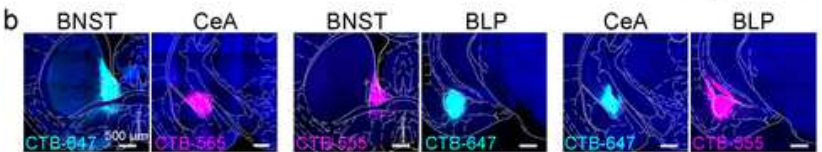

d

Proportion of $\mathrm{CTB}+\pi \mathrm{TH}+$ cells in DRN
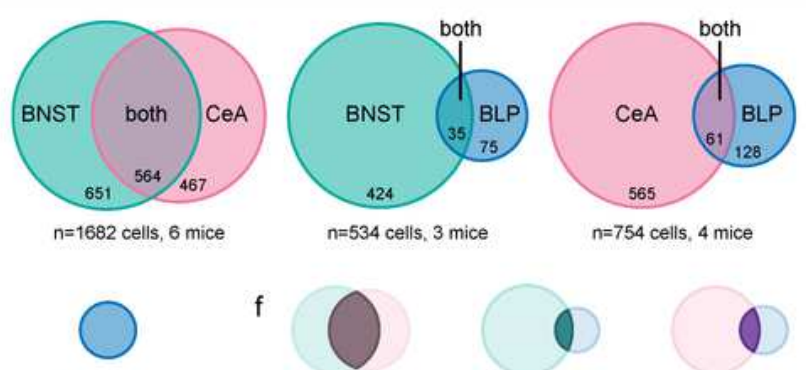

f

DRN ${ }^{\text {DAT }}$
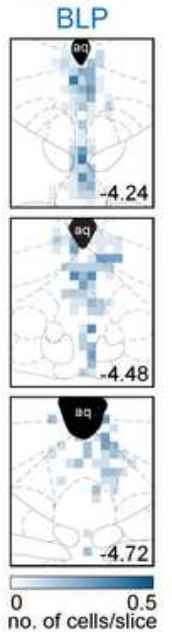
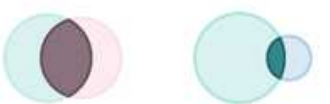

DRN ${ }^{\text {DAT_- }}$ BNST $\cap \mathrm{CEA}$
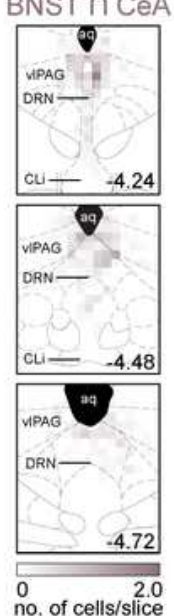
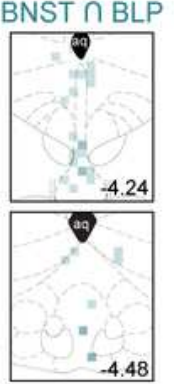

CEA $\cap$ BLP
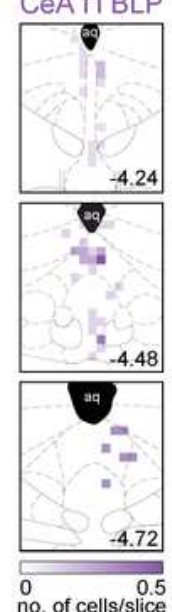

\section{Figure 2}

Collateralization of DRNDAT projections. a, The retrograde tracer cholera toxin subunit-B (CTB) conjugated to Alexa Fluor 555 (CTB-555, magenta) or Alexa-Fluor 647 (CTB-647, cyan) was injected into two downstream targets. b, Confocal images showing representative injection sites for dual BNST and CeA injections (left panels), BNST and BLP (center panels), and CeA and BLP (right panels). c, High magnification images of DRN cells expressing CTB-555 (magenta), CTB-647 (cyan), and TH (green) 
following injection into the BNST and CeA. White arrows indicate triple-labelled cells. d, Venn diagrams showing the proportion of $\mathrm{CTB}+/ \mathrm{TH}+$ cells in the DRN following dual injections placed in the BNST and CeA (left), BNST and BLP (center), or CeA and BLP (right). When injections were placed in the BNST and CeA, dual CTB-labelled TH+ cells constituted $46 \%$ of all BNST projectors and $55 \%$ of all CeA projectors. In contrast, when injections were placed in the BNST and BLP, or CeA and BLP, the proportion of duallabelled cells was considerably lower (7.6\% of BNST projectors and $9.7 \%$ of CeA projectors). e, Heatmaps indicating the relative density of $\mathrm{TH}+\mathrm{CTB}+$ cells throughout the $\mathrm{DRN} / \mathrm{CLi}$ for each projector population and $\mathrm{f}$, dual-labelled cells. Color intensity represents average number of cells per slice. The total number of $\mathrm{TH}+\mathrm{BNST}$ and CeA projectors per slice was similar ( $\mathrm{n}=27.9$ BNST projectors and $n=27.2$ CeA projectors per slice), whereas TH+ BLP projectors were significantly fewer in number ( $n=6.4$ BLP projectors per slice; Kruskal-Wallis statistic $=83.5, p<0.0001$; Dunn's post-hoc tests: BNST vs. CeA $p>0.05$, BNST vs BLP $p<0.001$, CeA vs BLP $p<0.001)$. TH+ BNST and CeA projectors, and dual-labelled cells, were broadly distributed throughout the DRN, vIPAG, and CLi, with a higher concentration in the dorsal aspect of the DRN, whereas BLP projectors tended to be relatively denser in ventral DRN/CLi. 
Figure 3
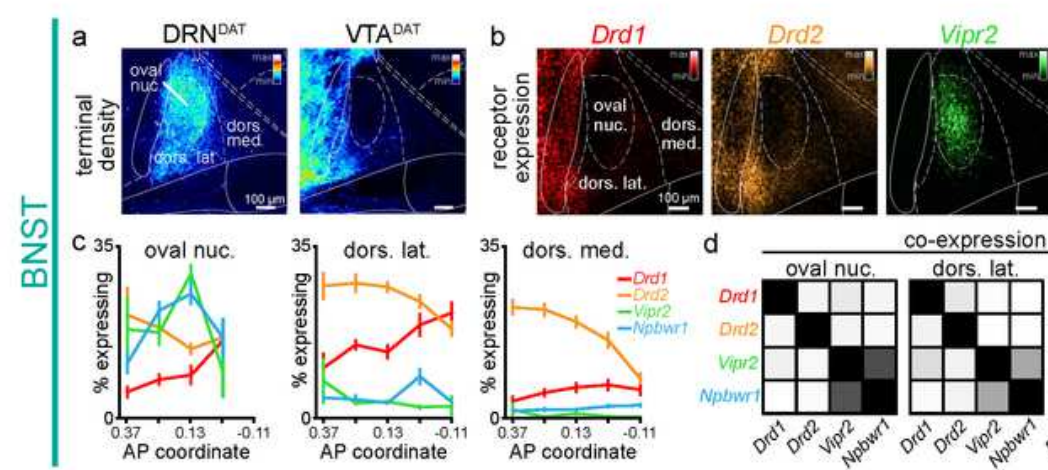

Nobwrit
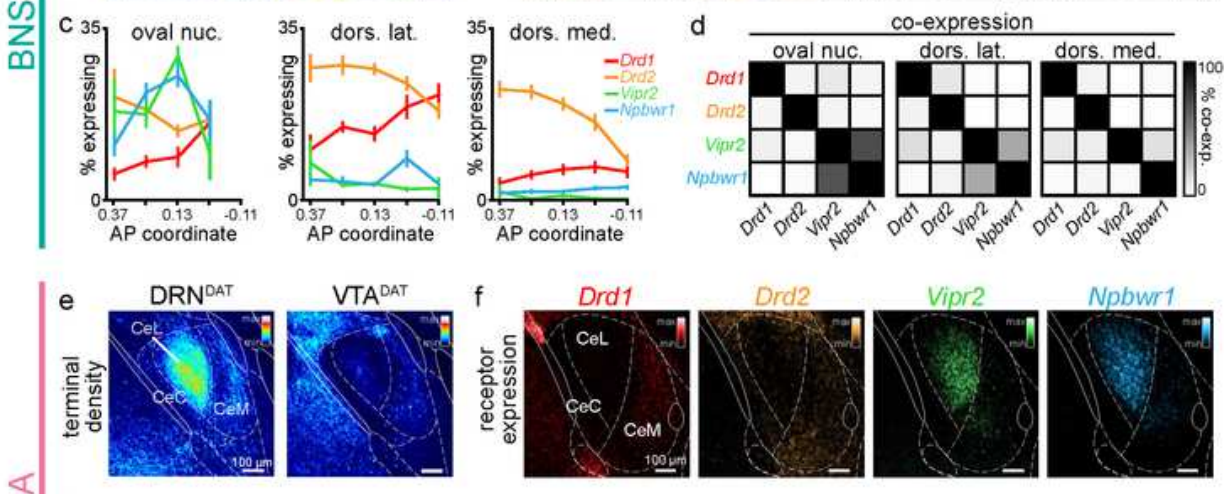

Nobwr1

()
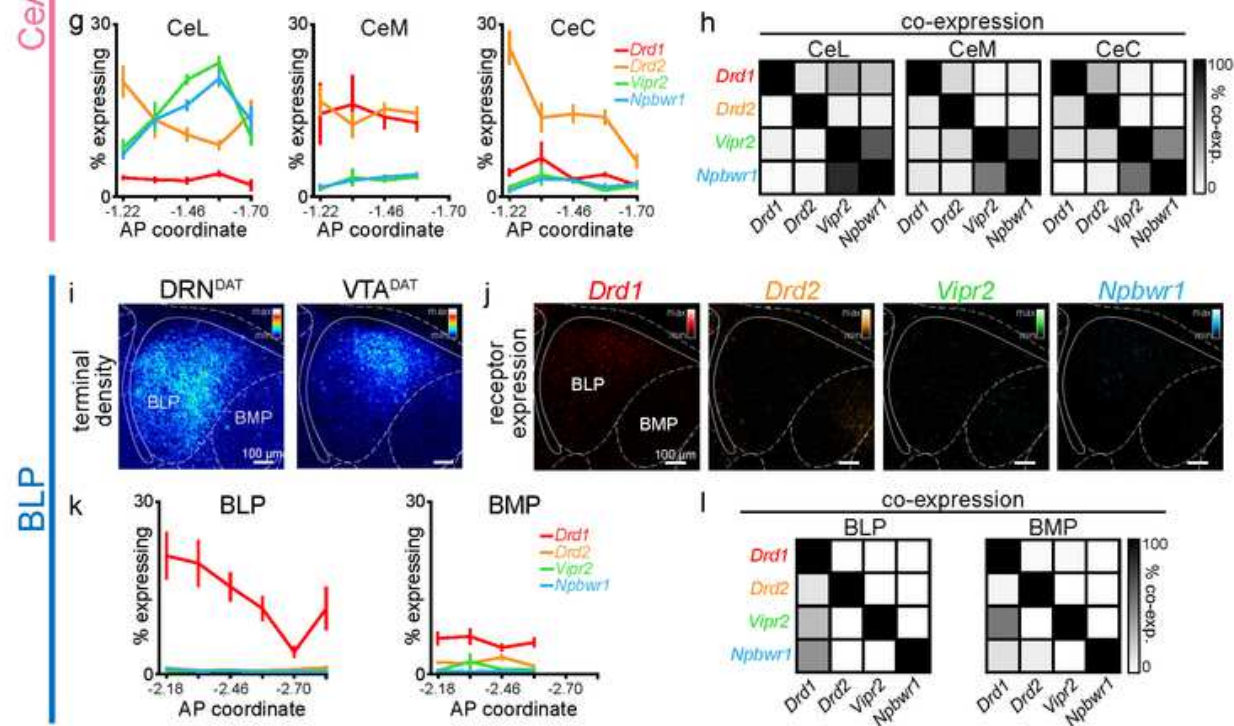

Nobwr1
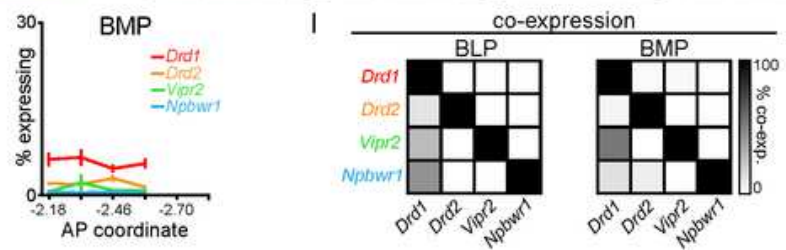

\section{Figure 3}

Spatial-segregation of dopamine and neuropeptide receptor populations within DRNDAT terminal fields.

a, Average image showing terminal density in the middle anteroposterior (AP) region of the BNST, following eYFP expression in DRNDAT (left) or VTADAT (right) neurons. b, Average images showing fluorescent puncta in the BNST indicating detection of Drd1 (red), Drd2 (yellow), Vipr2 (green), or Npbwr1 (blue) mRNA transcripts. c, Line graphs showing the percent of cells expressing each receptor ( $\geq 5$ puncta) 
across AP locations for the oval nucleus, dorsolateral BNST, and dorsomedial BNST (two-way ANOVA, oval nucleus: RNA probe $x$ AP interaction, $F 9,160=6.194, p<0.0001$, dorsolateral $B N S T$ : RNA probe $x$ AP interaction, $F 12,167=3.410, p=0.0002$, dorsomedial BNST: RNA probe $x$ AP interaction, $F 12,161=2.268$, $p=0.0110$ ). Drd1: $n=51,55,53$, Drd2: $n=52,55,53$, Vipr2: $n=37,39,37$, Npbwr1: $n=36,38,38$ sections, for oval nucleus, dorsolateral BNST, and dorsomedial BNST, respectively, from 4 mice. d, Matrices indicating overlap between mRNA-expressing cells: square color indicates the percent of cells expressing the gene in the column from within cells expressing the gene in the row. e, Average image showing terminal density in the middle AP region of the CeA, following eYFP expression in DRNDAT (left) or VTADAT (right) neurons. $\mathrm{f}$, Average images showing fluorescent puncta in the CeA indicating mRNA expression. $g$, Line graphs showing the $\%$ of cells expressing each receptor ( $\geq 5$ puncta) across AP locations for the CeL, CeM, and CeC (two-way ANOVA, CeL: RNA probe x AP interaction, F12,220=8.664, $p<0.0001$, CeM: main effect of RNA probe, F3,186=60.30, $p<0.0001, \mathrm{CeC}$ : RNA probe $x$ AP interaction, $F 12,218=4.883, p<0.0001)$. Drd1: $n=47,40,47$, Drd2: $n=70,55,70$, Vipr2: $n=65,57,63$, Npbwr1: $n=62,50,60$ sections, for CeL, CeM, and CeC, respectively, from 4 mice. h, Matrices indicating overlap between mRNA-expressing cells. i, Average image showing terminal density in the middle AP region of the BLP, following eYFP expression in DRNDAT (left) or VTADAT (right) neurons. j, Average images showing fluorescent puncta in the BLP indicating mRNA expression. $k$, Line graphs showing the percent of cells expressing each receptor ( $\geq 5$ puncta) across AP locations for the BLP and BMP (two-way ANOVA, BLP: RNA probe $x$ AP interaction, F15,176=2.165, $p=0.0091$, BMP: main effect of RNA probe, F3,141=56.92, $p<0.0001$ ). Drd1: $n=55,44$ Drd2: $n=59,46$ Vipr2: $n=41,33$ Npbwr1: $n=45,34$ sections, for BLP and BMP, respectively, from 4 mice. I, Matrices indicating overlap between mRNA-expressing cells. Line graphs show mean \pm SEM. 

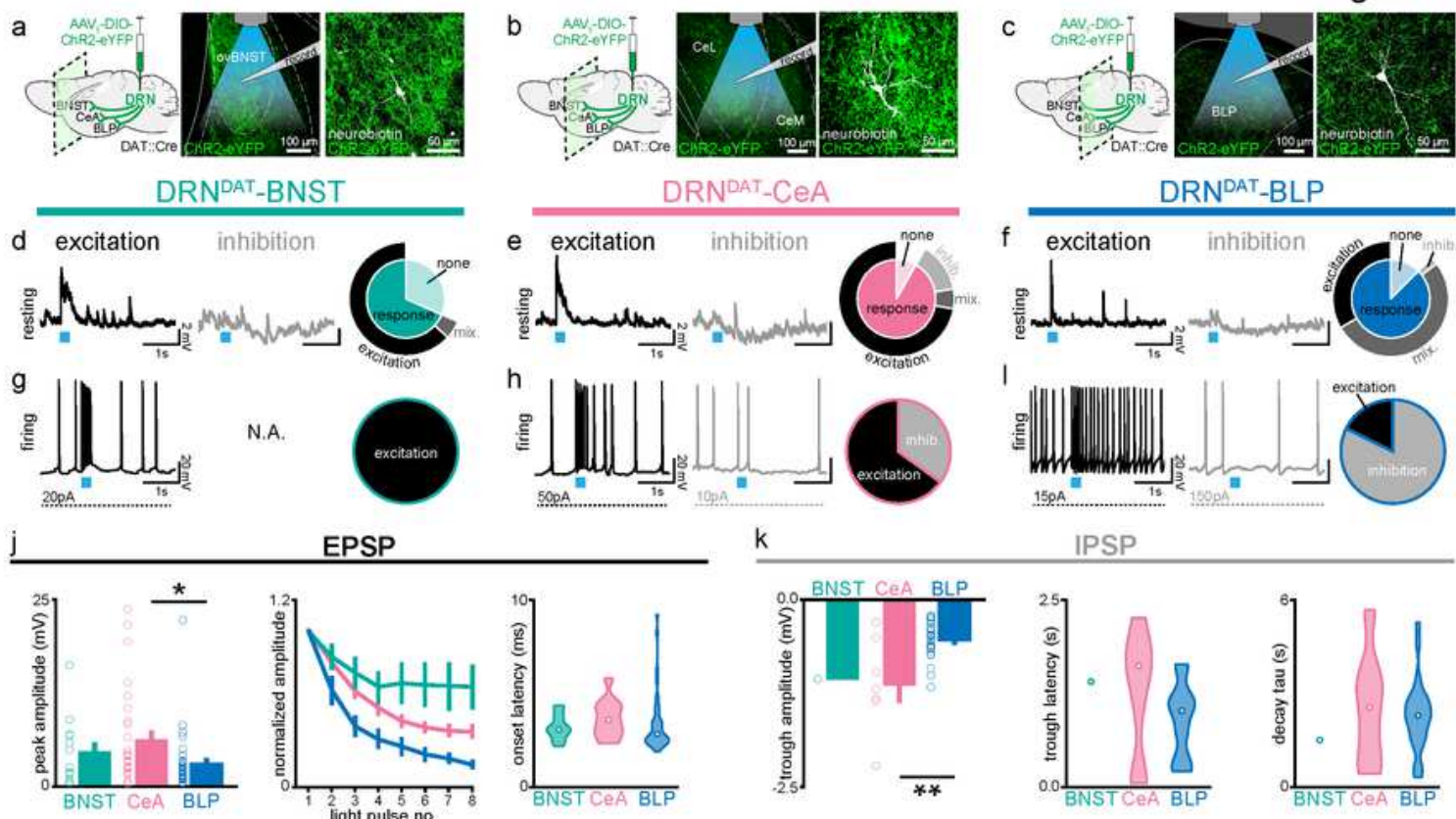

EPSP

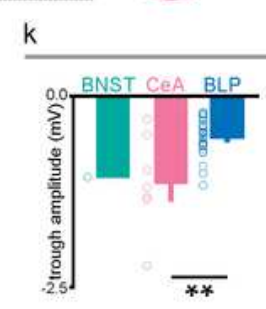

IPSP

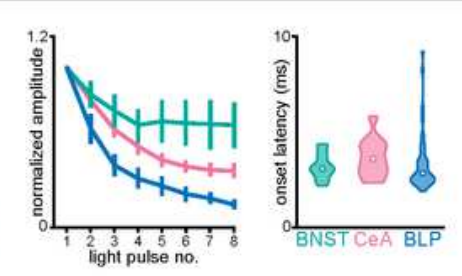

CeA
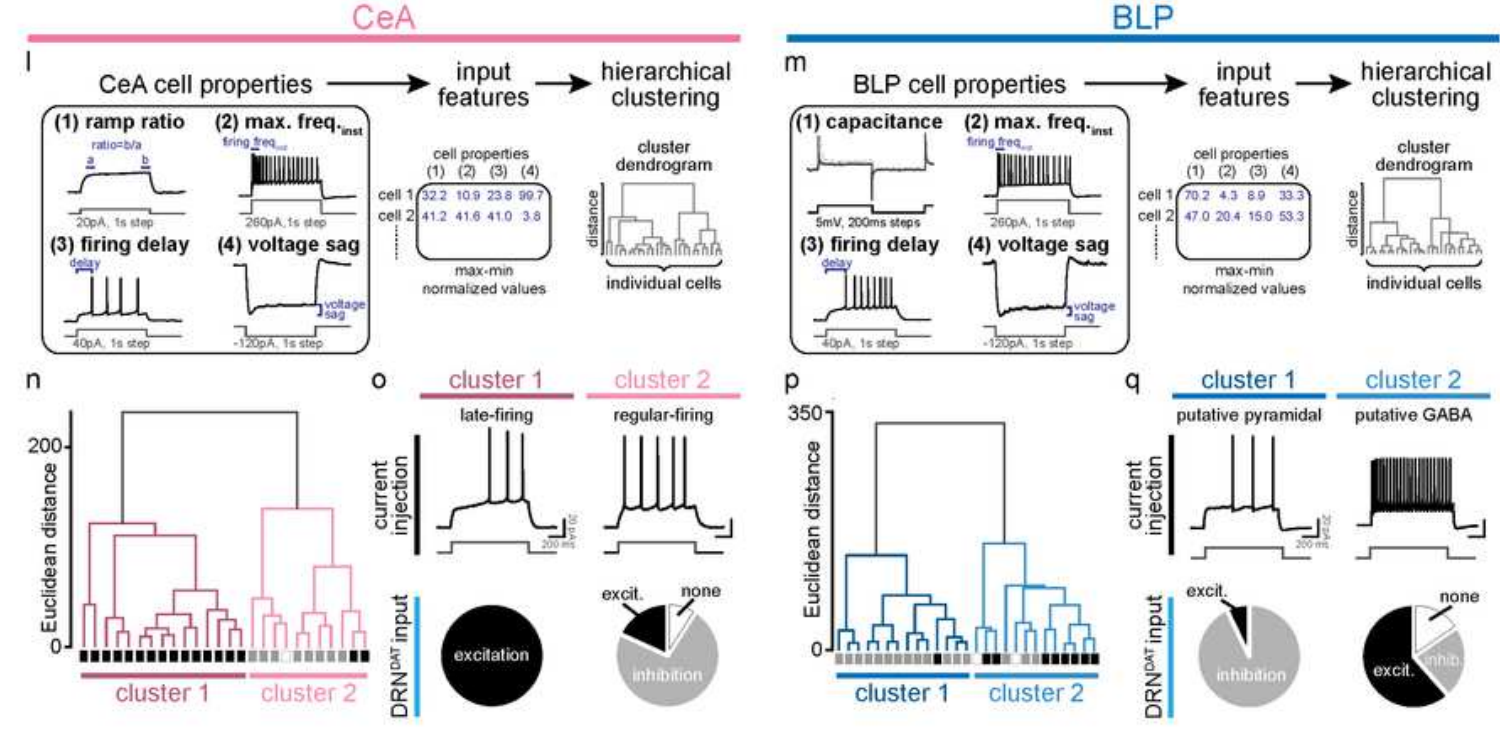

\section{Figure 4}

DRNDAT input influences downstream activity in a cell type-dependent manner. a, In mice expressing ChR2 in DRNDAT neurons, ex vivo electrophysiological recordings were made from the BNST, b, CeA, and c, BLP. d, Photostimulation of DRNDAT terminals with blue light (8 pulses delivered at $30 \mathrm{~Hz}$ ) evoked both excitatory and inhibitory responses at resting membrane potentials in the BNST, e, CeA, and f, BLP. Traces show single sweeps and pie charts indicate proportion of cells with no response ('none'), an EPSP only 
('excitation'), an IPSP only ('inhibition'), or a mixed response ('mix'). Recorded cells: BNST n=19, CeA $n=36$, $B L P n=48$, from 19 mice. $g$, When constant current was injected to elicit spontaneous firing, BNST cells responded to photostimulation with an increase in firing ('excitation'), while h, CeA and i, BLP cells responded with an increase or a decrease in firing ('inhibition'). Recorded cells: BNST $n=5$, CeA $n=20, B L P$ $n=17$. j, Properties of the optically-evoked excitatory post-synaptic potential (EPSP) at resting membrane potentials - left: peak amplitude (Kruskal-Wallis statistic $=6.790, p=0.0335$; Dunn's posts-hoc tests: CeA vs BLP $p=0.0378$ ); middle: change in amplitude across light pulses; right: violin plots showing distribution of onset latencies (white circle indicates median). $k$, Properties of the optically-evoked inhibitory postsynaptic potential (EPSP) at resting membrane potentials - left: trough amplitude (one-way ANOVA, $F 2,31=8.150, p=0.0014$, CeA vs BLP: ${ }^{\star \star} p=0.0014$ ); middle: violin plot showing latency to trough peak; right: violin plot showing tau for the current decay (white circle indicates median). Bar and line graphs display mean \pm SEM. ${ }^{*} p<0.05$, ${ }^{*} p<0.01$. I, Workflow for agglomerative hierarchical clustering of CeA neurons and $\mathrm{m}$, BLP neurons. Four baseline electrical properties were used as input features, following max-min normalization (see Methods) and Ward's method used to generate a cluster dendrogram, grouping cells based on Euclidean distance. n, Dendrogram for CeA cells indicating two major clusters, with their response to DRNDAT input indicated below each branch (excitation=black; inhibition=grey; no response=open). o, Upper panels: cluster 1 showed baseline properties typical of 'late-firing' neurons and cluster 2 showed baseline properties typical of 'regular-firing' neurons. Lower panels: pie charts showing the response of cells in each cluster to DRNDAT input. $p$, Dendrogram for BLP cells indicating two major clusters, with their response to DRNDAT input indicated below each branch (excitation=black; inhibition=grey; no response=open). q, Upper panels: cluster 1 showed baseline properties typical of pyramidal neurons and cluster 2 showed baseline properties typical of GABA interneurons. Lower panels: pie charts showing the response of cells in each cluster to DRNDAT input.

\section{Supplementary Files}

This is a list of supplementary files associated with this preprint. Click to download.

- SupplementaryFigureLegends.docx 